\title{
Transient three-dimensional contact problems: mortar method. Mixed methods and conserving integration
}

\author{
Christian Hesch • Peter Betsch
}

Received: 1 October 2010 / Accepted: 13 February 2011 / Published online: 17 April 2011

(C) Springer-Verlag 2011

\begin{abstract}
The present work deals with the development of an energy-momentum conserving method to unilateral contact constraints and is a direct continuation of a previous work (Hesch and Betsch in Comput Mech 2011, doi:10.1007/ s00466-011-0597-2) dealing with the NTS method. In this work, we introduce the mortar method and a newly developed segmentation process for the consistent integration of the contact interface. For the application of the energy-momentum approach to mortar constraints, we extend an approach based on a mixed formulation to the segment definition of the mortar constraints. The enhanced numerical stability of the newly proposed discretization method will be shown in several examples.
\end{abstract}

Keywords Contact - Energy methods · Large deformation · Mortar method

\section{Introduction}

The most common approach for the simulation of contact problems in the context of large deformations is the well known node-to-segment (NTS) method. This method is often preferred due to its simple implementation and has also been addressed in the precursor [16] of the present work. For a survey of actual developments in the field of NTS methods we refer to the textbooks written by Laursen [22] and Wriggers [35]. The collocation-type NTS method does not pass the patch test and exhibits poor convergence properties. In particular, the local errors at the contact region do not necessarily diminish with mesh refinement (see [7]).

C. Hesch $(\varangle) \cdot$ P. Betsch

Chair of Computational Mechanics, Department of Mechanical

Engineering, University of Siegen, Siegen, Germany

e-mail: christian.hesch@uni-siegen.de
To remedy this drawback, mortar formulations have been used for unilateral contact problems for several years. Originally developed in the context of domain decomposition problems (see [2] and for further details [21,27,33,34]), they are nowadays well established for contact problems. The extension to contact mechanics started with the application of the mortar method to two-dimensional contact problems, see McDevitt and Laursen [26], Yang et al. [37], Flemish et al. [9] and Fischer and Wriggers [8]. Three dimensional implementations have been developed in Puso and Laursen [28,29].

Energy-momentum schemes for non-linear elastodynamics have been developed in the beginning of the 1990s, starting with the work of Simo and Tarnow [31] and Simo et al. [32]. Further improvements have been achieved by Gonzalez $[10,12]$ for general non-linear systems and extended to systems subject to holonomic constraints by Gonzalez [11], see also Betsch and Steinman [4].

A first application of energy-momentum schemes to unilateral contact constraints within the concept of the NTS method can be found in Laursen and Chawla [6,23] and in Armero and Petöcz [1]. Further developments, exclusively within the framework of the NTS method, can be found in Laursen and Love [24], Hauret and Le Tallec [14] and Haikal and Hjelmstad [13]. A first application within the mortar framework to two-dimensional contact problems and three-dimensional domain decomposition problems can be found in Hesch and Betsch [15, 17].

For the construction of an energy-momentum method, we use mixed or reducible formulations, see Zienkiewicz et al. [38]. In particular, we apply a specific coordinate augmentation technique, originally introduced by Betsch and Uhlar [5] in the context of multibody dynamics. This concept has already been modified for the construction of an energy-momentum scheme within the framework of domain decomposition problems [17]. Furthermore, we will show 
the drawbacks of this formulation and consider major simplifications, reducing the numerical costs of the mortar formulation to the costs of the widely used NTS method. Although we sacrifice exact conservation of total energy due to the proposed simplification, we are able to algorithmically conserve at least both momentum maps.

An outline of the present work is as follows. Section 2 gives a short introduction to finite dimensional Hamiltonian systems subject to unilateral contact constraints. The mortar constraints and the necessary segmentation process will be shown in detail in Sect. 3. In Sect. 4 follows the introduction of mixed formulations and the reformulation of the mortar constraints in terms of invariants. The objectivity of the semidiscrete system will be shown in Sect. 5, followed by the energy conserving time discretization scheme and the mentioned simplifications in Sect. 5.1. Representative numerical examples are presented in Sect. 6. Eventually, conclusions are drawn in Sect. 7.

\section{Mechanical systems with unilateral holonomic constraints}

Without loss of generality we consider a two body contact problem, where the reference configuration of the bodies in contact is represented by the open sets $\Omega^{(i)}, i \in\{1,2\}$. The surfaces on the current boundary $\gamma^{(i)}=\varphi^{(i)}\left(\partial \Omega^{(i)}, t\right)$ of the bodies are subdivided as follows

$\gamma^{(i)}=\gamma_{u}^{(i)} \cup \gamma_{\sigma}^{(i)} \cup \gamma_{c}^{(i)}, \quad \gamma_{u}^{(i)} \cap \gamma_{\sigma}^{(i)} \cap \gamma_{c}^{(i)}=0$

where $\gamma_{u}^{(i)}$ denotes the Dirichlet boundary, $\gamma_{\sigma}^{(i)}$ the Neumann boundary and $\gamma_{c}^{(i)}$ the contact boundary of the respective body.

A comprehensive treatment of the governing equations can be found in our previous paper [?]. Here, we focus on a finite-dimensional mechanical system, subject to unilateral holonomic constraints. Within this framework, the augmented Hamiltonian $\mathcal{H}$, a function of the configuration vector $\boldsymbol{q}(t) \in \mathbb{R}^{n}$, the linear momentum $\boldsymbol{p}(t) \in \mathbb{R}^{n}$ and the Lagrange multipliers $\lambda(t) \in \mathbb{R}^{m}$ at time $t$ reads

$\mathcal{H}(\boldsymbol{q}, \boldsymbol{p}, \boldsymbol{\lambda})=T(\boldsymbol{p})+V_{\lambda}(\boldsymbol{q})$

where $T(\boldsymbol{p})$ denotes the total kinetic energy related to a nonsingular mass matrix $\boldsymbol{M} \in \mathbb{R}^{n} \times \mathbb{R}^{n}$

$T(\boldsymbol{p})=\frac{1}{2} \boldsymbol{p} \cdot \boldsymbol{M}^{-1} \boldsymbol{p}$

and $V_{\lambda}(\boldsymbol{q})$ denotes an augmented potential energy function, given by

$V_{\lambda}(\boldsymbol{q})=V(\boldsymbol{q})+\lambda \cdot \boldsymbol{\Phi}$

The potential energy function $V(\boldsymbol{q})$ describes the strain energy of the discretized bodies involved in a contact situation. Details concerning the strain energy function are given in standard textbooks (e.g. $[20,22])$. Note that we place no restrictions to the strain energy functions, neither to the constitutive law nor to the strain measures. Additionally, the Lagrange multipliers $\lambda$ are used to enforce $m$ holonomic constraints $\boldsymbol{\Phi}: \mathbb{R}^{n} \rightarrow \mathbb{R}^{m}$.

Throughout this paper, we use isoparametric displacement-based finite elements in space for the approximation of the configuration and its variation

$\boldsymbol{q}^{(i)}=\sum_{A \in \omega^{(i)}} N_{A}^{(i)} \boldsymbol{q}_{A}^{(i)}, \quad$ and $\delta \boldsymbol{q}^{(i)}=\sum_{A \in \omega^{(i)}} N_{A}^{(i)} \delta \boldsymbol{q}_{A}^{(i)}$

where $A \in \omega^{(i)}=\left\{1, \ldots, n_{\text {node }}^{(i)}\right\}$ defines a set of nodes and $n_{\text {node }}^{(i)}$ the corresponding total number of nodes of the body $i \in\{1,2\}$. For convenience, we introduce a subset $A \in \bar{\omega}^{(i)}=\left\{1, \ldots, n_{\text {surf }}^{(i)}\right\}$ of nodes on the respective contact interfaces $\gamma_{c}^{(i)}$ and the total number of constraints $n^{\text {con }}$. To deal with unilateral contact constraints $\boldsymbol{\Psi}$, which are subject to the classical Karush-Kuhn-Tucker conditions

$\Psi_{I} \leq 0, \quad \lambda_{I} \geq 0, \quad \lambda_{I} \Psi_{I}=0, \quad \forall i \in\left\{1, \ldots, n^{\text {con }}\right\}$

we replace the original constraints $\boldsymbol{\Psi}$ with the already introduced constraints $\boldsymbol{\Phi}$

$\Phi_{I}=\lambda_{I}-\max \left\{0, \lambda_{I}-c \Psi_{I}\right\}, \quad c>0$

For details on the corresponding active-set strategy see Hüeber and Wohlmuth [19], for details concerning the differentiability of the max-operator see Hintermueller et al. [18].

\subsection{Mortar formulation}

Once the actual contact boundary is determined, we can formulate the local balance of linear momentum across the interface (cf. [37])

$\boldsymbol{t}^{(1)} \mathrm{d} \gamma^{(1)}=-\boldsymbol{t}^{(2)} \mathrm{d} \gamma^{(2)}$

where $\boldsymbol{t}^{(i)}$ denotes the Cauchy tractions. Based on this balance principle we can postulate the virtual work of the contact forces $G^{c}$ (cf. [30])

$G^{c}=\sum_{i=1}^{2} \int_{\gamma_{c}^{(i)}} \boldsymbol{t}^{(i)} \cdot \delta \boldsymbol{q}^{(i)} \mathrm{d} \gamma^{(i)}=\int_{\gamma_{c}^{(1)}} \boldsymbol{t}^{(1)} \cdot\left[\delta \boldsymbol{q}^{(1)}-\delta \boldsymbol{q}^{(2)}\right] \mathrm{d} \gamma$

In correspondence with the literature, surface $\gamma_{c}^{(1)}$ is referred to as non-mortar surface, while the opposing surface is called mortar surface. In contrast to the NTS-method, which utilizes a point wise evaluation of the Cauchy tractions, we interpolate the tractions using the shape functions $N^{A}$ of the underlying discrete surface on the non-mortar side. Accordingly,

$\boldsymbol{t}^{(1)}=\sum_{A=\bar{\omega}^{(1)}} N^{A}\left(\boldsymbol{\xi}^{(1)}\right) \boldsymbol{t}_{A}^{(1)}$ 
Fig. 1 Three dimensional segmentation problem

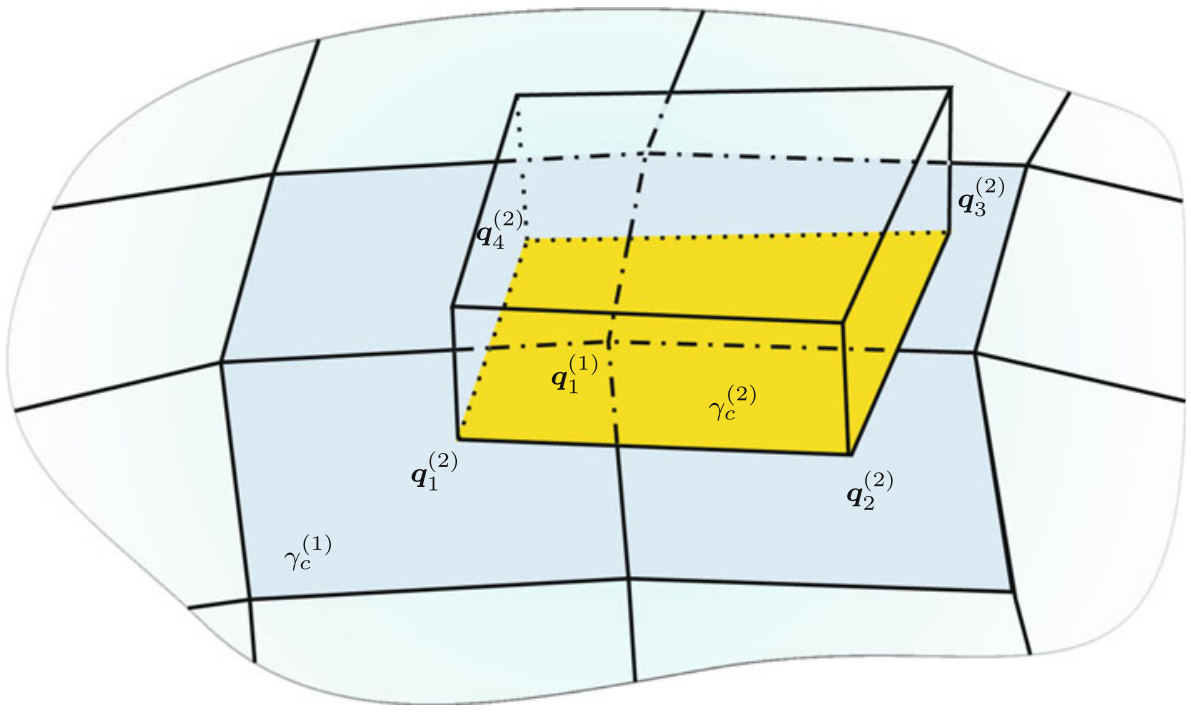

If we substitute (5) and (10) into (9) we obtain the discrete contact virtual work ${ }^{1}$

$G^{c}=\boldsymbol{n}^{B} \cdot \delta \boldsymbol{q}_{B}^{(1)}-\boldsymbol{n}^{C} \cdot \delta \boldsymbol{q}_{C}^{(2)}$

where the abbreviations

$\boldsymbol{n}^{B}=\int_{\gamma_{c}^{(1)}} \boldsymbol{t}_{A} N^{A}\left(\boldsymbol{\xi}^{(1)}\right) N^{B}\left(\boldsymbol{\xi}^{(1)}\right) \mathrm{d} \gamma, \quad$ and
$\boldsymbol{n}^{C}=\int_{\gamma_{c}^{(1)}} \boldsymbol{t}_{A} N^{A}\left(\boldsymbol{\xi}^{(1)}\right) N^{C}\left(\boldsymbol{\xi}^{(2)}\right) \mathrm{d} \gamma$

have been used. The Cauchy tractions are decomposed into the normal and the tangential part

$\boldsymbol{t}_{A}=\boldsymbol{t}_{A}^{N}+\boldsymbol{t}_{A}^{F}, \quad$ with $\boldsymbol{t}_{A}^{N}=\lambda_{A} \boldsymbol{v}, \quad \boldsymbol{t}^{F} \cdot \boldsymbol{v}=0$

where $\boldsymbol{v}$ denotes the unit outward normal vector of the surface $\gamma_{c}^{(1)}$. Since we restrict ourselves to frictionless sliding, we assume $\boldsymbol{t}^{F}=\mathbf{0}$. The mortar constraints in normal directions can now be written as follows

$\Psi^{A}=\boldsymbol{n}^{A B} \cdot \boldsymbol{q}_{B}^{(1)}-\boldsymbol{n}^{A C} \cdot \boldsymbol{q}_{C}^{(2)}$

where

$$
\begin{gathered}
\boldsymbol{n}^{A B}=\int_{\gamma_{c}^{(1)}} \boldsymbol{v} N^{A}\left(\boldsymbol{\xi}^{(1)}\right) N^{B}\left(\boldsymbol{\xi}^{(1)}\right) \mathrm{d} \gamma \quad \text { and } \\
\boldsymbol{n}^{A C}=\int_{\gamma_{c}^{(1)}} \boldsymbol{v} N^{A}\left(\boldsymbol{\xi}^{(1)}\right) N^{C}\left(\xi^{(2)}\right) \mathrm{d} \gamma
\end{gathered}
$$

\footnotetext{
${ }^{1}$ If convenient and unique, the summation convention is used.
}

are referred to as mortar integrals. The evaluation of the mortar integrals (15) is based on a segmentation process, described in detail in the following section.

\section{Mortar contact constraints}

The evaluation of the mortar integrals relies on the simultaneous integration of both dissimilarly discretized surfaces in contact. To deal with this matter, we subdivide both surfaces into segments and introduce a common parametrization based on triangular shape functions within each single segment. Then we apply Gauss integration and assemble the segment contributions into a global vector of constraints.

This section is organized as follows: In Sect. 3.1 we discuss the newly developed segmentation process for arbitrary curved surfaces. There are well-established segmentation procedures (see [30,35] and the references therein); we have to reconsider these procedures, since we assume the segments to be configuration dependent. The evaluation of the mortar constraints will be shown in Sect. 3.2, followed by the assembly procedure outlined in Sect. 3.3.

\subsection{Determination of the segments}

A typical situation is depicted in Fig. 1. The lower surface of the brick element (highlighted in yellow) on the mortar side and the corresponding portions of the four opposing surface elements on the non-mortar side are assumed to be in contact. The following outlines the algorithm for the segmentation process:

1. Loop over all nodes $\boldsymbol{q}_{I}^{(2)}$ on the mortar side.

Similar on the standard orthogonal projection technique known from the NTS method we determine the 


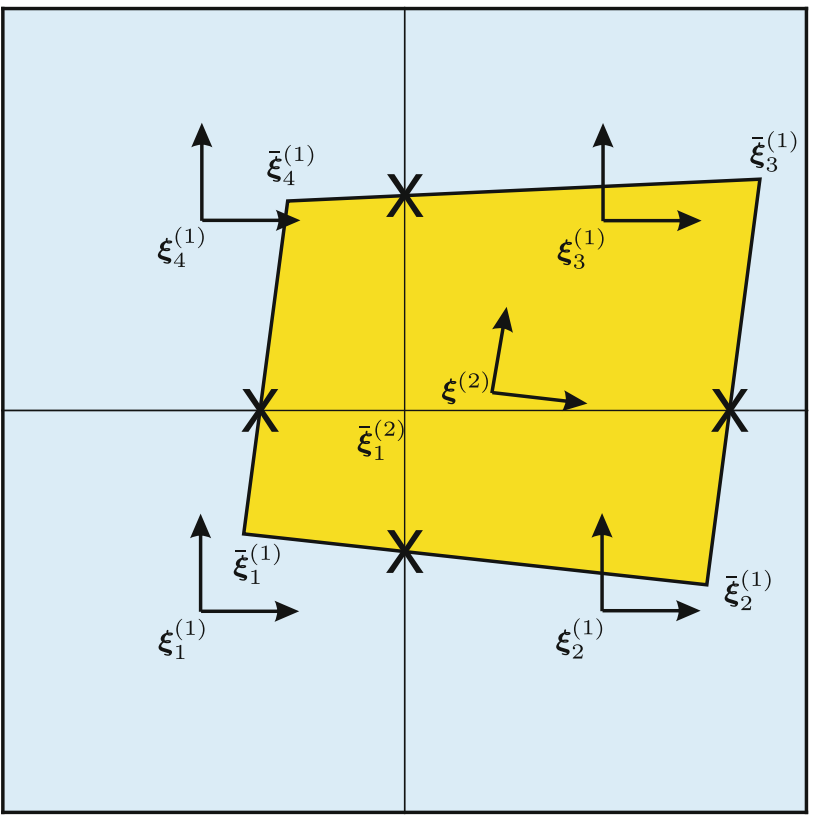

Fig. 2 Orthogonal projection of the vertices

convective coordinates $\bar{\xi}_{I}^{(1)}$ corresponding to the vertices $\boldsymbol{q}_{I}^{(2)}$ (see Fig. 2) by solving the non-linear equations

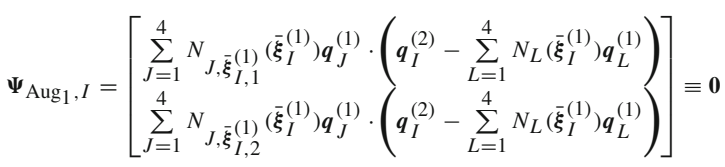

with respect to the convective coordinates using a standard Newton method. Here, $N_{J, \bar{\xi}_{I, \alpha}^{(1)}}\left(\bar{\xi}_{I}^{(1)}\right)$ denotes the derivative of the shape function with respect to $\bar{\xi}_{I, \alpha}^{(1)}$.

2. Loop over all nodes $\boldsymbol{q}_{I}^{(1)}$ on the non-mortar side.

Once again, we use the orthogonal projection technique to determine the convective coordinates $\bar{\xi}_{I}^{(2)}$ corresponding to the relevant nodes on the mortar side (see $\bar{\xi}_{1}^{(2)}$ in Fig. 2). To deal with arbitrary curved surfaces, we project the nodes to the projected surfaces defined by the nodes $\overline{\boldsymbol{q}}_{I}^{(1)}=\sum_{L=1}^{4} N_{L}\left(\overline{\boldsymbol{\xi}}_{I}^{(1)}\right) \boldsymbol{q}_{L}^{(1)}$ instead of the original surface defined by the nodes $\boldsymbol{q}_{I}^{(2)}$. Once again, we use a Newton method to solve the non-linear equations

$$
\begin{aligned}
& \boldsymbol{\Psi}_{\mathrm{Aug}_{2}, I} \\
& =\left[\begin{array}{c}
\sum_{J=1}^{4} N_{J, \overline{\boldsymbol{\xi}}_{I, 1}^{(2)}}\left(\overline{\boldsymbol{\xi}}_{I}^{(2)}\right) \overline{\boldsymbol{q}}_{J}^{(1)} \cdot\left(\boldsymbol{q}_{I}^{(1)}-\sum_{K=1}^{4} N_{K}\left(\overline{\boldsymbol{\xi}}_{I}^{(2)}\right) \overline{\boldsymbol{q}}_{K}^{(1)}\right) \\
\sum_{J=1}^{4} N_{J, \overline{\boldsymbol{\xi}}_{I, 2}^{(2)}}\left(\overline{\boldsymbol{\xi}}_{I}^{(2)}\right) \overline{\boldsymbol{q}}_{J}^{(1)} \cdot\left(\boldsymbol{q}_{I}^{(1)}-\sum_{K=1}^{4} N_{K}\left(\overline{\boldsymbol{\xi}}_{I}^{(2)}\right) \overline{\boldsymbol{q}}_{K}^{(1)}\right)
\end{array}\right] \equiv \mathbf{0}
\end{aligned}
$$

with respect to the convective coordinates $\bar{\xi}_{I}^{(2)}$.



Fig. 3 Determination of the intersections

3. Loop over all edges on the non-mortar side.

To determine the projected intersections between the edges (see the crosses in Fig. 2), we create a list of all edges of all surface elements on the mortar side and span on each edge, corresponding to the nodes $\boldsymbol{q}_{K}^{(1)}, K \in\{1,2\}$, a surface using a normal field, ${ }^{2}$ defined by $\boldsymbol{d}_{K}$ at both nodes $\boldsymbol{q}_{K}^{(1)}$. Then we create a second list of all edges of the projected mesh. A specific line on the projected mesh can be determined using the projected nodes $\overline{\boldsymbol{q}}_{J}^{(1)}=\sum_{L=1}^{4} N_{L}\left(\overline{\boldsymbol{\xi}}_{J}^{(1)}\right) \boldsymbol{q}_{L}^{(1)}, J \in\{1,2\}$. At last, we search for the intersection (see Fig. 3) between each projected line and each possible surface. The corresponding convective coordinates $\tilde{\xi}_{I, 1}$ and $\tilde{\xi}_{I, 2}$ (as well as $\tilde{\xi}_{I, 3}$ which is not needed in the sequel) follow from

$$
\begin{aligned}
\boldsymbol{\Psi}_{\operatorname{Aug}_{3}, I}= & \sum_{I=1}^{2} \hat{N}_{J}\left(\tilde{\xi}_{I, 1}\right) \overline{\boldsymbol{q}}_{J}^{(1)} \\
& -\sum_{K=1}^{2} \hat{N}_{K}\left(\tilde{\xi}_{I, 2}\right)\left(\boldsymbol{q}_{K}^{(1)}+\boldsymbol{d}_{K}^{(1)} \tilde{\xi}_{I, 3}\right) \equiv \mathbf{0}
\end{aligned}
$$

Note that in the above considerations the shape functions $\hat{N}_{J}\left(\tilde{\xi}_{I, i}\right), i \in\{1,2\}$ on the edges are one dimensional (see Fig. 3).

4. Delaunay triangularization of each element on the mortar side.

Based on the results of the first three steps we apply a Delaunay triangularization as shown in Fig. 4. Note that several constraints (i.e. specification of nodes, which must be connected) have to be predetermined. As shown in Fig. 4, two segments $\mathrm{Seg}_{1}$ and $\mathrm{Seg}_{2}$ have been located in the first square of Fig. 2.

For later use and guided by previous developments in [?] we introduce a global vector of coordinates $\boldsymbol{f}$, collecting all convective coordinates, determined by (16), (17) and (18).

2 Different definitions of a normal vector on a discrete surface are possible, see e.g. Yang et al. [37]. We use an averaged normal composed of the normals of the adjacent elements. 
Fig. 4 Segmentation based on a Delaunay triangularization

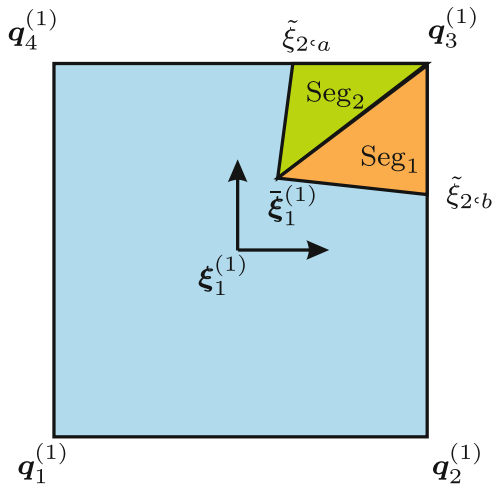

\subsection{Segment wise evaluation of the mortar constraints}

After we have located all segments, we calculate the segment contributions to the mortar constraints (14). Therefore we introduce for each segment a linear transformation $\boldsymbol{\eta} \rightarrow \tilde{\boldsymbol{\xi}}_{\text {seg }}^{(i)}$ via

$\tilde{\boldsymbol{\xi}}_{\mathrm{seg}}^{(i)}(\boldsymbol{\eta})=\sum_{K=1}^{3} M^{K}(\boldsymbol{\eta}) \boldsymbol{\xi}_{\mathrm{seg}, K}^{(i)}$

where $\boldsymbol{\xi}_{\mathrm{seg}, K}^{(i)}$ denote the convective coordinates determined in Sect. 3.1. For each segment we specify the associated convective coordinates and collect them in the set $\eta_{\text {conv }}=$ $\left\{\boldsymbol{f}_{\text {seg }}\right\}=\left\{\boldsymbol{\xi}_{\text {seg }, K}^{(i)}\right\}, i \in\{1,2\}, K \in\{1,2,3\}$. In accordance with the results of the segmentation algorithm, linear triangular shape functions $M^{K}$ are used. The approximations (5) and (10) can now be recast in the form

$\boldsymbol{t}_{\mathrm{seg}}^{(1)}=\sum_{\kappa} N^{\kappa}\left(\tilde{\boldsymbol{\xi}}_{\mathrm{seg}}^{(1)}(\boldsymbol{\eta})\right) \boldsymbol{t}_{\kappa}$

$\boldsymbol{q}_{\mathrm{seg}}^{(1)}=\sum_{\beta} N^{\beta}\left(\tilde{\boldsymbol{\xi}}_{\mathrm{seg}}^{(1)}(\boldsymbol{\eta})\right) \boldsymbol{q}_{\beta}^{(1)}$

$\boldsymbol{q}_{\mathrm{seg}}^{(2)}=\sum_{\zeta} N^{\zeta}\left(\tilde{\boldsymbol{\xi}}_{\mathrm{seg}}^{(2)}(\boldsymbol{\eta})\right) \boldsymbol{q}_{\zeta}^{(2)}$

For the application of a numerical quadrature rule, the Jacobian

$J_{\text {seg }}=\left\|\boldsymbol{a}_{1}\left(\tilde{\boldsymbol{\xi}}_{\text {seg }}^{(1)}(\boldsymbol{\eta})\right) \times \boldsymbol{a}_{2}\left(\tilde{\boldsymbol{\xi}}_{\text {seg }}^{(1)}(\boldsymbol{\eta})\right)\right\| \operatorname{det}(D \boldsymbol{\xi}(\boldsymbol{\eta}))$

is required, where the tangential vectors $\boldsymbol{a}_{\alpha}$ are calculated via

$\boldsymbol{a}_{\alpha}=\sum_{\kappa} N_{, \xi_{\alpha}}^{\kappa}\left(\tilde{\boldsymbol{\xi}}_{\mathrm{seg}}^{(1)}(\boldsymbol{\eta})\right) \boldsymbol{q}_{\kappa}^{(1)}$

Based on the tangential vectors, we specify a unit normal vector $\boldsymbol{v}$

$\boldsymbol{v}=\frac{\boldsymbol{a}_{1}\left(\tilde{\boldsymbol{\xi}}_{\mathrm{seg}}^{(1)}(\boldsymbol{\eta})\right) \times \boldsymbol{a}_{2}\left(\tilde{\boldsymbol{\xi}}_{\mathrm{seg}}^{(1)}(\boldsymbol{\eta})\right)}{\left\|\boldsymbol{a}_{1}\left(\tilde{\boldsymbol{\xi}}_{\mathrm{seg}}^{(1)}(\boldsymbol{\eta})\right) \times \boldsymbol{a}_{2}\left(\tilde{\boldsymbol{\xi}}_{\mathrm{seg}}^{(1)}(\boldsymbol{\eta})\right)\right\|}$
The we cut the norm from both, the Jacobian and the normal vector and receive

$\bar{J}_{\text {seg }}=\operatorname{det}(D \xi(\eta)), \quad \overline{\boldsymbol{v}}=\boldsymbol{a}_{1}\left(\tilde{\boldsymbol{\xi}}_{\text {seg }}^{(1)}(\boldsymbol{\eta})\right) \times \boldsymbol{a}_{2}\left(\tilde{\boldsymbol{\xi}}_{\text {seg }}^{(1)}(\boldsymbol{\eta})\right)$

To prevent expensive calculations we propose at this point a simplification and assume that $\overline{\boldsymbol{v}}$ remains constant in each segment, i.e. we evaluate the normal vector at a specific, constant position within the segment. ${ }^{3}$ Then we can rewrite the constraints on segment level as follows

$\Psi_{\mathrm{seg}}^{\kappa}=\overline{\boldsymbol{v}} \cdot\left[\bar{n}^{\kappa \beta} \boldsymbol{q}_{\beta}^{(1)}-\bar{n}^{\kappa \zeta} \boldsymbol{q}_{\zeta}^{(2)}\right]$

using the mortar integrals

$$
\begin{aligned}
& \bar{n}^{\kappa \beta}\left(\boldsymbol{f}_{\mathrm{seg}}\right)=\int_{\triangle} N^{\kappa}\left(\tilde{\boldsymbol{\xi}}_{\mathrm{seg}}^{(1)}(\boldsymbol{\eta})\right) N^{\beta}\left(\tilde{\boldsymbol{\xi}}_{\mathrm{seg}}^{(1)}(\boldsymbol{\eta})\right) \overline{\boldsymbol{J}}_{\mathrm{seg}} \mathrm{d} \eta \\
& \bar{n}^{\kappa \zeta}\left(\boldsymbol{f}_{\mathrm{seg}}\right)=\int_{\triangle} N^{\kappa}\left(\tilde{\boldsymbol{\xi}}_{\mathrm{seg}}^{(1)}(\boldsymbol{\eta})\right) N^{\zeta}\left(\tilde{\boldsymbol{\xi}}_{\mathrm{seg}}^{(2)}(\boldsymbol{\eta})\right) \bar{J}_{\mathrm{seg}} \mathrm{d} \eta
\end{aligned}
$$

which we evaluate using a standard Gauss quadrature (cf. $[17,27]$ ).

\subsection{Assembly of the mortar constraints}

Once we have determined all segment contributions, we have to assemble the mortar constraints. Therefore, we arrange the constraint functions in a global vector of constraints $\Psi(q)$ in correspondence to the Lagrange multipliers, which are related to the nodal points on the non-mortar side

$$
\sum_{A \in \bar{\omega}^{(1)}} \lambda_{A} \Psi^{A}(\boldsymbol{q}, \boldsymbol{f})=\boldsymbol{\lambda} \cdot \boldsymbol{\Psi}(\boldsymbol{q}, \boldsymbol{f})
$$

Each segment in turn corresponds to a pair of elements $e_{1} \in$ $\bar{\epsilon}^{(1)}$, where $\bar{\epsilon}^{(1)}$ denotes the set of elements on the contact surface $\gamma_{c}^{(1)}$, and $e_{2} \in \bar{\epsilon}^{(2)}$ on the contact surface $\gamma_{c}^{(2)}$. Since each constraint will be assembled out of a variable number of segments, where each triangular segment relies on the four

\footnotetext{
${ }^{3}$ It is important to note that the normal vector still depends on the current configuration of the surface, only its relative position on the surface remains constant.
} 
vertices of $e_{1}$ with local node number $\kappa \in\{1, \ldots, 4\}$, we need a connection between the local node numbers and the global location within the vector of constraints $\Psi(\boldsymbol{q})$. Therefore we introduce a location array LM (see [15]), so that $A=\operatorname{LM}\left(\kappa, e_{1}\right)$ and use this location array for the assembly of the segment contributions

$$
\begin{aligned}
\boldsymbol{\Psi}_{\text {mortar }}(\boldsymbol{q}, \boldsymbol{f}) & =\underset{e_{1} \in \bar{\epsilon}^{(1)}}{\mathbf{A}} \boldsymbol{\Psi}_{e_{1}}(\boldsymbol{q}, \boldsymbol{f}) \\
& =\underset{e_{1} \in \bar{\epsilon}^{(1)}}{\mathbf{A}} \bigcup_{\mathrm{seg}} \boldsymbol{\Psi}_{e_{1}, \mathrm{seg}}\left(\boldsymbol{q}_{\mathrm{seg}}, \boldsymbol{f}_{\mathrm{seg}}\right) \\
& =\mathbf{e}_{e_{1} \in \bar{\epsilon}^{(1)}} \bigcup_{\mathrm{seg}}\left[\begin{array}{c}
\Psi_{e_{1}, \mathrm{seg}}\left(\boldsymbol{q}_{\mathrm{seg}}, \boldsymbol{f}_{\mathrm{seg}}\right) \\
\vdots \\
\Psi_{e_{1}, \mathrm{seg}}^{\kappa=4}\left(\boldsymbol{q}_{\mathrm{seg}}, \boldsymbol{f}_{\mathrm{seg}}\right)
\end{array}\right]
\end{aligned}
$$

where $\mathbf{A}$ denotes the standard assembly operator (see [20]) and $\boldsymbol{q}_{\text {seg }}$ is defined by the set of relevant vectors $\eta_{\text {seg }}=$ $\left\{\boldsymbol{q}_{\mathrm{seg}}\right\}=\left\{\boldsymbol{q}_{I}^{(1)}, \boldsymbol{q}_{J}^{(2)}\right\}, \forall I, J \in[1, \ldots, 4]$.

\section{Reformulation of the constraints}

Regarding Cauchy's representation theorem, we can rewrite (cf. Sect. 4.1) the constraints in terms of invariants. For the later application of the concept of a discrete gradient to conserve the total energy, we have to reformulate the constraints in terms of invariants, which are at most quadratic. Therefore, as outlined in Hesch and Betsch [? 17], we replace the normal vector for each segment by augmented coordinates $\boldsymbol{d}_{\text {seg }}$ in the mortar constraints

$$
\begin{aligned}
& \Psi_{\mathrm{seg}}^{\kappa}\left(\boldsymbol{q}_{\mathrm{seg}}, \boldsymbol{f}_{\mathrm{seg}}, \boldsymbol{d}_{\mathrm{seg}}\right) \\
& \quad=\boldsymbol{d}_{\mathrm{seg}} \cdot\left[\bar{n}^{\kappa \beta}\left(\boldsymbol{f}_{\mathrm{seg}}\right) \boldsymbol{q}_{\beta}^{(1)}-\bar{n}^{\kappa \zeta}\left(\boldsymbol{f}_{\mathrm{seg}}\right) \boldsymbol{q}_{\zeta}^{(2)}\right] .
\end{aligned}
$$

Note that the additional constraints

$$
\boldsymbol{\Psi}_{\mathrm{seg}}^{\mathrm{normal}}=\left[\begin{array}{c}
\boldsymbol{d}_{\mathrm{seg}} \cdot \boldsymbol{a}_{1} \\
\boldsymbol{d}_{\mathrm{seg}} \cdot \boldsymbol{a}_{2} \\
\boldsymbol{d}_{\mathrm{seg}} \cdot \boldsymbol{d}_{\mathrm{seg}}-\left\|\boldsymbol{a}_{1} \times \boldsymbol{a}_{2}\right\|^{2}
\end{array}\right]
$$

are necessary to determine the actual value of the augmented coordinates.

\subsection{Reformulation in terms of invariants}

Similar to the approach in Hesch and Betsch [?] we rewrite the mortar constraints (31) in terms of invariants. Therefore, we introduce the following sets

$$
\begin{aligned}
& \overline{\mathbb{S}}\left(\eta_{\text {aug }}\right)=\left\{\left(\boldsymbol{q}_{I}^{(1)}-\boldsymbol{q}_{1}^{(1)}\right) \cdot\left(\boldsymbol{q}_{J}^{(i)}-\boldsymbol{q}_{1}^{(1)}\right), i \in\{1,2\}, I, J \in\{1,2,3,4\}\right\} \\
& \tilde{\mathbb{S}}\left(\eta_{\text {aug }}\right)=\left\{\left(\boldsymbol{q}_{I}^{(i)}-\boldsymbol{q}_{1}^{(1)}\right) \cdot \boldsymbol{d}_{\text {seg }}, i \in\{1,2\}, I \in\{1,2,3,4\}\right\} \\
& \mathbb{S}\left(\eta_{\text {aug }}\right)=\left\{\boldsymbol{d}_{\text {seg }} \cdot \boldsymbol{d}_{\text {seg }}\right\} \\
& \hat{\mathbb{S}}\left(\eta_{\text {aug }}\right)=\left\{\boldsymbol{f}_{\text {seg }}\right\}
\end{aligned}
$$

and define a vector of possible invariants

$\boldsymbol{\pi}=\left[\begin{array}{c}\overline{\boldsymbol{\pi}}^{(i)} \in \overline{\mathbb{S}}\left(\eta_{\text {aug }}\right) \\ \tilde{\boldsymbol{\pi}}^{(i)} \in \tilde{\mathbb{S}}\left(\eta_{\text {aug }}\right) \\ \stackrel{\circ}{\boldsymbol{\pi}} \in \mathbb{S}\left(\eta_{\text {aug }}\right) \\ \hat{\boldsymbol{\pi}} \in \hat{\mathbb{S}}\left(\eta_{\text {aug }}\right)\end{array}\right]$

Note that we have chosen $\boldsymbol{q}_{1}^{(1)}$ such that all terms with $I=1$ in (33) vanish. Other choices using a different number of invariants are possible. To rewrite (31) in terms of the invariants (34) we rearrange the constraints as follows

$$
\begin{aligned}
\Psi_{\mathrm{seg}}^{\kappa}\left(\boldsymbol{q}_{\mathrm{seg}}, \boldsymbol{f}_{\mathrm{seg}}, \boldsymbol{d}_{\mathrm{seg}}\right)= & \bar{n}^{\kappa \beta}\left(\boldsymbol{f}_{\mathrm{seg}}\right) \boldsymbol{d}_{\mathrm{seg}} \cdot \boldsymbol{q}_{\beta}^{(1)} \\
& -\bar{n}^{\kappa \zeta}\left(\boldsymbol{f}_{\mathrm{seg}}\right) \boldsymbol{d}_{\mathrm{seg}} \cdot \boldsymbol{q}_{\zeta}^{(2)}
\end{aligned}
$$

As shown in Puso [27], linear momentum can not be exactly conserved due to the inexact numerical evaluation of the mortar integrals. This drawback can be removed by assuming that

$$
\sum_{\beta} \bar{n}^{\kappa \beta} \boldsymbol{q}_{1}^{(1)}-\sum_{\zeta} \bar{n}^{\kappa \zeta} \boldsymbol{q}_{1}^{(1)}=\mathbf{0}
$$

holds exactly. Note that the evaluation of the mortar integrals (28) by means of quadrature rules violates condition (36) in general. Inserting (36) in (35) yields

$$
\begin{aligned}
& \Psi_{\text {seg }}^{\kappa}\left(\boldsymbol{q}_{\mathrm{seg}}, \boldsymbol{f}_{\mathrm{seg}}, \boldsymbol{d}_{\mathrm{seg}}\right) \\
& \quad=\bar{n}^{\kappa \beta}\left(\boldsymbol{f}_{\mathrm{seg}}\right)\left(\boldsymbol{q}_{\beta}^{(1)}-\boldsymbol{q}_{1}^{(1)}\right) \cdot \boldsymbol{d}_{\mathrm{seg}}-\bar{n}^{\kappa \zeta}\left(\boldsymbol{f}_{\mathrm{seg}}\right)\left(\boldsymbol{q}_{\zeta}^{(2)}-\boldsymbol{q}_{1}^{(1)}\right) \cdot \boldsymbol{d}_{\mathrm{seg}}
\end{aligned}
$$

Applying the sets of invariants (33) the mortar constraints (31) can now be written as follows

$\Psi_{\mathrm{seg}}^{\kappa}(\boldsymbol{\pi})=\bar{n}^{\kappa \beta}(\hat{\boldsymbol{\pi}}) \tilde{\pi}_{\beta}^{(1)}-\bar{n}^{\kappa \zeta}(\hat{\boldsymbol{\pi}}) \tilde{\pi}_{\zeta}^{(2)}$

The additional constraints (32) used to determine the actual values of the augmented coordinates $\boldsymbol{d}_{\text {seg }}$ have also to be rewritten in terms of invariants

$\boldsymbol{\Psi}_{\mathrm{seg}}^{\mathrm{normal}}(\boldsymbol{\pi})=\left[\begin{array}{c}\sum_{I=1}^{4} N_{I, \xi_{1}^{(1)}} \tilde{\pi}_{I}^{(1)} \\ \sum_{I=1}^{4} N_{I, \xi_{2}^{(1)}} \tilde{\pi}_{I}^{(1)} \\ \pi-K\end{array}\right]$

with

$$
\begin{aligned}
K= & {\left[\left(N_{I, \xi_{1}^{(1)}} N_{J, \xi_{1}^{(1)}} \bar{\pi}_{I, J}^{(1)}\right)\left(N_{I, \xi_{2}^{(1)}} N_{J, \xi_{2}^{(1)}} \bar{\pi}_{I, J}^{(1)}\right)\right.} \\
& \left.-\left(N_{I, \xi_{1}^{(1)}} N_{J, \xi_{2}^{(1)}} \bar{\pi}_{I, J}^{(1)}\right)^{2}\right]
\end{aligned}
$$


The corresponding reformulation of the constraints in (16), (17) and (18) can be found in Appendix A. Note that the constraints $\boldsymbol{\Psi}_{\mathrm{Aug}_{3}}$ in (18) have to be modified as follows

$$
\boldsymbol{\Psi}_{\text {Aug }}^{\mathrm{ed}_{I} J}=\left[\begin{array}{c}
\boldsymbol{d}_{1}^{(1)} \cdot\left[\sum_{I=1}^{2} \hat{N}_{I}\left(\tilde{\xi}_{1}\right) \overline{\boldsymbol{q}}_{I}^{(1)}-\sum_{J=1}^{2} \hat{N}_{J}\left(\tilde{\xi}_{2}\right)\left(\boldsymbol{q}_{J}^{(1)}+\boldsymbol{d}_{J}^{(1)} \tilde{\xi}_{3}\right)\right] \\
\left(\boldsymbol{q}_{1}^{(1)}-\boldsymbol{q}_{2}^{(1)}\right) \cdot\left[\sum_{I=1}^{2} \hat{N}_{I}\left(\tilde{\xi}_{1}\right) \overline{\boldsymbol{q}}_{I}^{(1)}-\sum_{J=1}^{2} \hat{N}_{J}\left(\tilde{\xi}_{2}\right)\left(\boldsymbol{q}_{J}^{(1)}+\boldsymbol{d}_{J}^{(1)} \tilde{\xi}_{3}\right)\right] \\
\left(\overline{\boldsymbol{q}}_{1}^{(1)}-\overline{\boldsymbol{q}}_{2}^{(1)}\right) \cdot\left[\sum_{I=1}^{2} \hat{N}_{I}\left(\tilde{\xi}_{1}\right) \overline{\boldsymbol{q}}_{I}^{(1)}-\sum_{J=1}^{2} \hat{N}_{J}\left(\tilde{\xi}_{2}\right)\left(\boldsymbol{q}_{J}^{(1)}+\boldsymbol{d}_{J}^{(1)} \tilde{\xi}_{3}\right)\right]
\end{array}\right]
$$

As will be shown in the following, the premultiplication of the original, nonlinear constraints using a local basis composed of $\boldsymbol{d}_{J}^{(1)},\left(\boldsymbol{q}_{1}^{(1)}-\boldsymbol{q}_{2}^{(1)}\right)$ and $\left(\overline{\boldsymbol{q}}_{1}^{(1)}-\overline{\boldsymbol{q}}_{2}^{(1)}\right)$ is necessary for the conservation of angular momentum. ${ }^{4}$

At last, we collect all constraints in one global vector

$\boldsymbol{\Psi}(\boldsymbol{\pi}(\boldsymbol{q}, \boldsymbol{f}, \boldsymbol{d}))=\left[\begin{array}{l}\boldsymbol{\Psi}_{\text {mortar }}(\boldsymbol{\pi}(\boldsymbol{q}, \boldsymbol{f}, \boldsymbol{d})) \\ \boldsymbol{\Psi}_{\mathrm{normal}(\boldsymbol{\pi}(\boldsymbol{q}, \boldsymbol{f}, \boldsymbol{d}))} \\ \boldsymbol{\Psi}_{\mathrm{Aug}_{1}(\boldsymbol{\pi}(\boldsymbol{q}, \boldsymbol{f}, \boldsymbol{d}))} \\ \boldsymbol{\Psi}_{\mathrm{Aug}_{2}(\boldsymbol{\pi}(\boldsymbol{q}, \boldsymbol{f}, \boldsymbol{d}))} \\ \boldsymbol{\Psi}_{\mathrm{Aug}_{3}}(\boldsymbol{\pi}(\boldsymbol{q}, \boldsymbol{f}, \boldsymbol{d}))\end{array}\right]$

Remark The original constraint (27) can be rewritten in terms of invariants, which are at most cubic

$\breve{\boldsymbol{\pi}}_{\mathrm{seg}}=\left[\begin{array}{c}\left(\boldsymbol{a}_{1} \times \boldsymbol{a}_{2}\right) \cdot\left(\boldsymbol{q}_{\beta}^{(1)}-\boldsymbol{q}_{1}^{(1)}\right) \\ \left(\boldsymbol{a}_{1} \times \boldsymbol{a}_{2}\right) \cdot\left(\boldsymbol{q}_{\zeta}^{(2)}-\boldsymbol{q}_{1}^{(1)}\right) \\ \boldsymbol{f}_{\mathrm{seg}}\end{array}\right], \quad \forall \beta, \zeta \in\{1, \ldots, 4\}$

The segment contributions to the mortar constraints based on (43) can now be written as follows

$\breve{\Psi}_{\text {seg }}^{\kappa}(\pi)=\bar{n}^{\kappa \beta}(\breve{\boldsymbol{\pi}}) \breve{\pi}_{\beta}^{(1)}-\bar{n}^{\kappa \zeta}(\breve{\boldsymbol{\pi}}) \breve{\pi}_{\zeta}^{(2)}$

After the assembly procedure (see (30)) we obtain the reformulated mortar constraints $\breve{\boldsymbol{\Psi}}_{\text {mortar }}(\breve{\boldsymbol{\pi}}(\boldsymbol{q}, \boldsymbol{f}, \boldsymbol{d}))$. Note, however, that cubic invariants prevent the application of the concept of a discrete gradient in the sense of Gonzalez [11].

\subsection{Frame indifference of the constraints}

According to Noether's theorem, conservation laws are related to the invariance properties of the system. In particular, conservation of linear and angular momentum can be linked to the invariance of the Hamiltonian under translations and rotations, assuming the absence of external forces. With regard to (4) we postulate the invariance of the strain energy function $V(\boldsymbol{q})$ (cf. [3]) and concentrate our investigations on the constraint functions. To verify the frame indifference, rigid body motions of the form

$\boldsymbol{q}_{I, \mathrm{seg}}^{\sharp}=\boldsymbol{c}+\boldsymbol{Q q _ { I , \mathrm { seg } }}$

$\overline{4}$ Other local bases are possible. are considered, where $c \in \mathbb{R}^{3}$ is a constant vector and $\boldsymbol{Q} \in$ $S O(3)$ is a rotation tensor. Due to the definition of the sets in (33) and the demonstrated reformulation of the segment contributions in terms of invariants (38) we can state that

$$
\begin{aligned}
& \Psi_{\mathrm{seg}}^{\kappa}\left(\boldsymbol{\pi}\left(\boldsymbol{c}+\boldsymbol{Q} \boldsymbol{q}_{I, \mathrm{seg}}, \boldsymbol{f}_{\mathrm{seg}}, \boldsymbol{d}_{\mathrm{seg}}\right)\right) \\
& \quad-\Psi_{\mathrm{seg}}^{\kappa}\left(\boldsymbol{\pi}\left(\boldsymbol{q}_{\mathrm{seg}}, \boldsymbol{f}_{\mathrm{seg}}, \boldsymbol{Q}^{T} \boldsymbol{d}_{\mathrm{seg}}\right)\right)=0
\end{aligned}
$$

Next, we substitute $\boldsymbol{c}=\epsilon \boldsymbol{\mu}$ and $\boldsymbol{Q}=\boldsymbol{I}$, where $\boldsymbol{I}$ denotes the unity matrix, $\mu \in \mathbb{R}^{3}$ is constant and $\epsilon \in \mathbb{R}$ arbitrary. Accordingly, we receive for the translational part

$$
\begin{aligned}
0 & =\left.\frac{\mathrm{d}}{\mathrm{d} \epsilon}\right|_{\epsilon=0} \Psi_{\mathrm{seg}}^{\kappa}\left(\boldsymbol{\pi}\left(\boldsymbol{q}_{I, \mathrm{seg}}+\epsilon \boldsymbol{\mu}, \boldsymbol{f}_{\mathrm{seg}}, \boldsymbol{d}_{\mathrm{seg}}\right)\right) \\
& \left.=D \Psi_{\mathrm{seg}}^{\kappa}(\boldsymbol{\pi})\right) \cdot \sum_{I} \partial_{\boldsymbol{q}_{I, \mathrm{seg}}} \boldsymbol{\pi}\left(\boldsymbol{q}_{\mathrm{seg}}, \boldsymbol{f}_{\mathrm{seg}}, \boldsymbol{d}_{\mathrm{seg}}\right) \cdot \boldsymbol{\mu}=0
\end{aligned}
$$

For the rotational part, we substitute $c=\mathbf{0}$ and $Q=$ $\exp (\epsilon \hat{\boldsymbol{\mu}})$, where $\exp (\epsilon \hat{\boldsymbol{\mu}}) \in S O(3)$ denotes the exponential map of a skew-symmetric tensor $\hat{\mu}$, which can be associated with an axial vector $\boldsymbol{\mu} \in \mathbb{R}^{3}$, so that $\hat{\boldsymbol{\mu}} \boldsymbol{a}=\boldsymbol{\mu} \times \boldsymbol{a}$ for any $\boldsymbol{a} \in \mathbb{R}^{3}$ and receive

$$
\begin{aligned}
& 0=\left.\frac{\mathrm{d}}{\mathrm{d} \epsilon}\right|_{\epsilon=0} \Psi_{\mathrm{seg}}^{\kappa}\left(\boldsymbol{\pi}\left(\exp (\epsilon \hat{\boldsymbol{\mu}}) \boldsymbol{q}_{I, \mathrm{seg}}, \boldsymbol{f}_{\mathrm{seg}}, \boldsymbol{d}_{\mathrm{seg}}\right)\right) \\
& \quad-\Psi_{\mathrm{seg}}^{\kappa}\left(\boldsymbol{\pi}\left(\boldsymbol{q}_{\mathrm{seg}}, \boldsymbol{f}_{\mathrm{seg}}, \exp (-\epsilon \hat{\boldsymbol{\mu}}) \boldsymbol{d}_{\mathrm{seg}}\right)\right) \\
&=D\left.\Psi_{\mathrm{seg}}^{\kappa}(\boldsymbol{\pi})\right) \cdot \sum_{I}\left[\partial_{\boldsymbol{q}_{I, \mathrm{seg}}} \pi\left(\boldsymbol{q}_{\mathrm{seg}}, \boldsymbol{f}_{\mathrm{seg}}, \boldsymbol{d}_{\mathrm{seg}}\right) \cdot\left(\boldsymbol{\mu} \times \boldsymbol{q}_{I, \mathrm{seg}}\right)\right. \\
&\left.+\partial_{\boldsymbol{d}_{\mathrm{seg}}} \pi\left(\boldsymbol{q}_{\mathrm{seg}}, \boldsymbol{f}_{\mathrm{seg}}, \boldsymbol{d}_{\mathrm{seg}}\right) \cdot\left(\boldsymbol{\mu} \times \boldsymbol{d}_{\mathrm{seg}}\right)\right]
\end{aligned}
$$

Since we rewrite the additional constraints (32), (16), (17) and (18) in terms of the same invariants, analogues properties are also valid for them.

\section{Equations of motion}

Regarding the Hamiltonian (2), the constrained semi-discrete system under consideration can be recast in the form

$$
\begin{aligned}
& \dot{z}=\mathbb{J} \nabla_{z} \mathcal{H}(z, \lambda) \\
& \mathbf{0}=\nabla_{\lambda} \mathcal{H}(z, \lambda)
\end{aligned}
$$

Here, $\mathbb{J}$ denotes the canonical skew symmetric matrix

$\mathbb{J}=\left[\begin{array}{cc}\mathbf{0} & \mathbf{1} \\ -\mathbf{1} & \mathbf{0}\end{array}\right]$

and $z=[\boldsymbol{q}, \boldsymbol{p}]$ the canonical phase space variables. Concerning the augmentation technique introduced in Sect. 4, the corresponding equations of motion take the form

$$
\begin{aligned}
\dot{z} & =\mathbb{J} \nabla_{z} \mathcal{H}(z, \boldsymbol{f}, \boldsymbol{d}, \boldsymbol{\lambda}) \\
\mathbf{0} & =\left[\begin{array}{l}
\nabla_{\boldsymbol{f}} \mathcal{H}(\boldsymbol{z}, \boldsymbol{f}, \boldsymbol{d}, \boldsymbol{\lambda}) \\
\nabla_{\boldsymbol{d}} \mathcal{H}(\boldsymbol{z}, \boldsymbol{f}, \boldsymbol{d}, \boldsymbol{\lambda}) \\
\nabla_{\boldsymbol{\lambda}} \mathcal{H}(\boldsymbol{z}, \boldsymbol{f}, \boldsymbol{d}, \boldsymbol{\lambda})
\end{array}\right]
\end{aligned}
$$


For the semi-discrete system at hand, various constants of motion exist (cf. [25, Chapter 3.3]). First, we consider the components of the momentum maps

$G(z)=\boldsymbol{\mu} \cdot \sum_{A \in \omega} \boldsymbol{p}_{A}, \quad G(z)=\boldsymbol{\mu} \cdot \sum_{A \in \omega} \boldsymbol{q}_{A} \times \boldsymbol{p}_{A}$

and show that

$\frac{\mathrm{d}}{\mathrm{d} t} G(z)=\nabla_{z} G(z) \cdot \dot{z}=\nabla_{z} G(z) \cdot \rrbracket \nabla_{z} \mathcal{H}(\boldsymbol{z}, \boldsymbol{f}, \boldsymbol{d}, \boldsymbol{\lambda}) \equiv \mathbf{0}$

Again, $\mu \in \mathbb{R}^{3}$ denotes a constant vector. Since we are not interested in the strain energy function (cf. [3]), we consider only the contributions of the reformulated active constraints. For the total linear momentum (52) 1 follows

$$
\begin{aligned}
\frac{\mathrm{d}}{\mathrm{d} t} G(\boldsymbol{z}) & =\boldsymbol{\mu} \cdot \sum_{A \in \bar{\omega}} \dot{\boldsymbol{p}}_{A} \\
& =\lambda \cdot D \boldsymbol{\Psi}(\boldsymbol{\pi}) \cdot \sum_{A \in \bar{\omega}}\left(\partial_{\boldsymbol{q}_{A}} \boldsymbol{\pi}(\boldsymbol{q}, \boldsymbol{d}, \boldsymbol{f})\right) \cdot \boldsymbol{\mu}=0
\end{aligned}
$$

where use of (47) has been made. Similarly, for the total angular momentum $(52)_{2}$ follows

$$
\begin{aligned}
\frac{\mathrm{d}}{\mathrm{d} t} G(z)= & \boldsymbol{\mu} \cdot \sum_{A \in \omega}\left(\dot{\boldsymbol{q}}_{A} \times \boldsymbol{p}_{A}+\boldsymbol{q}_{A} \times \dot{\boldsymbol{p}}\right) \\
= & \boldsymbol{\mu} \cdot \sum_{A \in \omega}\left(\boldsymbol{M}^{-1} \boldsymbol{p}_{A} \times \boldsymbol{p}_{A}-\boldsymbol{q}_{A}\right. \\
& \times\left(\partial_{\boldsymbol{q}_{A}} \boldsymbol{\pi}(\boldsymbol{q}, \boldsymbol{f}, \boldsymbol{d})\right)^{T} \nabla_{\boldsymbol{\pi}} \boldsymbol{\Psi}(\boldsymbol{\pi}) \cdot \boldsymbol{\lambda} \\
= & -\boldsymbol{\mu} \cdot \sum_{A \in \bar{\omega}} \boldsymbol{q}_{A} \times\left(\partial_{\boldsymbol{q}_{A}} \boldsymbol{\pi}(\boldsymbol{q}, \boldsymbol{f}, \boldsymbol{d})\right)^{T} \nabla_{\boldsymbol{\pi}} \boldsymbol{\Psi}(\boldsymbol{\pi}) \cdot \boldsymbol{\lambda} \\
= & \lambda \cdot D \boldsymbol{\Psi}(\boldsymbol{\pi}) \cdot \sum_{A \in \bar{\omega}}\left(\partial_{\boldsymbol{q}_{A}} \boldsymbol{\pi}(\boldsymbol{q}, \boldsymbol{f}, \boldsymbol{d})\right) \cdot \hat{\boldsymbol{q}}_{A} \boldsymbol{\mu}
\end{aligned}
$$

With regard to (48) we can state that

$$
\begin{array}{r}
D \boldsymbol{\Psi}(\boldsymbol{\pi}) \cdot\left[\sum_{A \in \bar{\omega}}\left(\partial_{\boldsymbol{q}_{A}} \boldsymbol{\pi}(\boldsymbol{q}, \boldsymbol{d}, \boldsymbol{f})\right) \cdot \hat{\boldsymbol{q}}_{A} \boldsymbol{\mu}\right. \\
\left.-\sum_{B \in \omega_{\mathrm{seg}}}\left(\partial_{\boldsymbol{d}_{B}} \boldsymbol{\pi}(\boldsymbol{q}, \boldsymbol{d}, \boldsymbol{f})\right) \cdot \hat{\boldsymbol{d}}_{B} \boldsymbol{\mu}\right]=\mathbf{0}
\end{array}
$$

where $\omega_{\text {seg }}$ denotes the set of all segments. Insertion in (55) together with $(51)_{3}$ yields

$$
\begin{aligned}
& \frac{\mathrm{d}}{\mathrm{d} t} G(z)=\lambda \cdot D \Psi(\pi) \cdot \sum_{B \in \omega_{\mathrm{seg}}}\left(\partial_{\boldsymbol{d}_{B}} \pi(\boldsymbol{q}, \boldsymbol{d}, \boldsymbol{f})\right) \cdot \hat{\boldsymbol{d}}_{B} \boldsymbol{\mu} \\
& \quad=0
\end{aligned}
$$

As before, only the contributions of the constraints have been considered. This demonstrates, that the frame-invariance is a necessary condition for the conservation of the momentum maps, which is in agreement with Noether's theorem. The last constant of motion considered here is the Hamiltonian itself, representing the total energy of the system

$G(z, d, f)=\mathcal{H}(z, f, d, \lambda)$

The invariance of the Hamiltonian with respect to time is a consequence of the skew-symmetry in (53). Due to the augmented coordinates, we have to consider the additional terms in (51) as well

$$
\begin{aligned}
\frac{\mathrm{d}}{\mathrm{d} t} G(z, \boldsymbol{d}, \boldsymbol{f})= & \nabla_{z} \mathcal{H}(\boldsymbol{z}, \boldsymbol{f}, \boldsymbol{d}, \boldsymbol{\lambda}) \dot{z}+\nabla_{\boldsymbol{f}} \mathcal{H}(z, \boldsymbol{f}, \boldsymbol{d}, \boldsymbol{\lambda}) \dot{\boldsymbol{f}} \\
& +\nabla_{\boldsymbol{d}} \mathcal{H}(\boldsymbol{z}, \boldsymbol{f}, \boldsymbol{d}, \boldsymbol{\lambda}) \dot{\boldsymbol{d}} \\
= & \nabla_{z} \mathcal{H}(z, \boldsymbol{f}, \boldsymbol{d}, \boldsymbol{\lambda}) \mathbb{J} \nabla_{z} \mathcal{H}(z, \boldsymbol{f}, \boldsymbol{d}, \boldsymbol{\lambda}) \\
& +\nabla_{\boldsymbol{d}} \mathcal{H}(\boldsymbol{z}, \boldsymbol{f}, \boldsymbol{d}, \boldsymbol{\lambda}) \dot{\boldsymbol{d}} \\
& +\nabla_{\boldsymbol{f}} \mathcal{H}(\boldsymbol{z}, \boldsymbol{f}, \boldsymbol{d}, \boldsymbol{\lambda}) \dot{\boldsymbol{f}} \\
= & 0
\end{aligned}
$$

representing the consistency condition of the Hamiltonian.

\subsection{Discretization in time}

To solve the semidiscrete system at hand, we have to implement an appropriate time stepping scheme. Three approaches for the discretization in time with different degrees of complexity are considered below.

1. The most complex approach rests on the configuration dependency of the mortar integrals. For a typical time step $\left[t_{n}, t_{n+1}\right]$ of length $\Delta t$ the equations of motion (51) can be recast in the form ${ }^{5}$

$$
\begin{aligned}
& \boldsymbol{q}_{n+1}-\boldsymbol{q}_{n}=\Delta t \boldsymbol{M}^{-1} \boldsymbol{p}_{n+\frac{1}{2}} \\
& \boldsymbol{p}_{n+1}-\boldsymbol{p}_{n}=-\Delta t \bar{\nabla} V\left(\boldsymbol{q}_{n}, \boldsymbol{q}_{n+1}\right) \\
& \quad-\Delta t\left(D_{1} \boldsymbol{\pi}\left(\boldsymbol{q}_{n+\frac{1}{2}}, \boldsymbol{d}_{n+\frac{1}{2}}, \boldsymbol{f}_{n+\frac{1}{2}}\right)\right)^{T} \overline{\bar{\nabla}}_{\boldsymbol{\pi}} \boldsymbol{\Psi}\left(\boldsymbol{\pi}_{n}, \boldsymbol{\pi}_{n+1}\right) \cdot \boldsymbol{\lambda}_{n+1} \\
& \mathbf{0}=\left(D_{2} \boldsymbol{\pi}\left(\boldsymbol{q}_{n+\frac{1}{2}}, \boldsymbol{d}_{n+\frac{1}{2}}, \boldsymbol{f}_{n+\frac{1}{2}}\right)\right)^{T} \overline{\bar{\nabla}}_{\boldsymbol{\pi}} \boldsymbol{\Psi}\left(\boldsymbol{\pi}_{n}, \boldsymbol{\pi}_{n+1}\right) \cdot \boldsymbol{\lambda}_{n+1} \\
& \mathbf{0}=\left(D_{3} \boldsymbol{\pi}\left(\boldsymbol{q}_{n+\frac{1}{2}}, \boldsymbol{d}_{n+\frac{1}{2}}, \boldsymbol{f}_{n+\frac{1}{2}}\right)\right)^{T} \overline{\bar{\nabla}}_{\boldsymbol{\pi}} \boldsymbol{\Psi}\left(\boldsymbol{\pi}_{n}, \boldsymbol{\pi}_{n+1}\right) \cdot \boldsymbol{\lambda}_{n+1} \\
& \mathbf{0}=\boldsymbol{\Psi}\left(\boldsymbol{\pi}\left(\boldsymbol{q}_{n+1}, \boldsymbol{d}_{n+1}, \boldsymbol{f}_{n+1}\right)\right)
\end{aligned}
$$

Here, $(\bullet)_{n+\frac{1}{2}}=\frac{1}{2}\left((\bullet)_{n+1}+(\bullet)_{n}\right)$ denotes a mid-point evaluation and $\bar{\nabla} V\left(\boldsymbol{q}_{n}, \boldsymbol{q}_{n+1}\right)$ is the discrete gradient of the strain energy function as proposed in Betsch and Steinmann [3]. The equivariant discrete gradient of the

\footnotetext{
${ }^{5}$ Here, $D_{1-3}$ denotes the derivative with respect to the $1-3$ slot.
} 
constraints $\overline{\bar{\nabla}}_{\pi} \boldsymbol{\Psi}\left(\boldsymbol{\pi}_{n}, \boldsymbol{\pi}_{n+1}\right)$ consists on the one hand of the segment contributions (see Sect. 3.3)

$$
\begin{aligned}
& \overline{\bar{\nabla}}_{\pi} \Psi_{\mathrm{seg}}^{\kappa}\left(\boldsymbol{\pi}_{n}, \boldsymbol{\pi}_{n+1}\right)=\nabla \Psi_{\mathrm{seg}}^{\kappa}\left(\boldsymbol{\pi}_{n+\frac{1}{2}}\right) \\
& +\frac{\Psi_{\mathrm{seg}}^{\kappa}\left(\boldsymbol{\pi}_{n+1}\right)-\Psi_{\mathrm{seg}}^{\kappa}\left(\boldsymbol{\pi}_{n}\right)-\nabla \Psi_{\mathrm{seg}}^{\kappa}\left(\pi_{n+\frac{1}{2}}\right) \Delta \pi}{\|\pi\|^{2}} \Delta \pi
\end{aligned}
$$

where $\Delta \boldsymbol{\pi}=\boldsymbol{\pi}_{n+1}-\boldsymbol{\pi}_{n}$. On the other hand, $\overline{\bar{\nabla}}_{\pi}$ $\boldsymbol{\Psi}\left(\boldsymbol{\pi}_{n}, \boldsymbol{\pi}_{n+1}\right)$ consists of the contributions of the reformulated constraints (42) $2-5$, using the same vector of invariants and the same definition for the discrete gradient.

2. A tremendous decrease of the size and the complexity of the system can be achieved by evaluating the convective coordinates $f$ only at time $t_{n}$. The equations of motion can now be recast in the form

$$
\begin{aligned}
& \boldsymbol{q}_{n+1}-\boldsymbol{q}_{n}=\Delta t \boldsymbol{M}^{-1} \boldsymbol{p}_{n+\frac{1}{2}} \\
& \boldsymbol{p}_{n+1}-\boldsymbol{p}_{n}=-\Delta t \bar{\nabla} V\left(\boldsymbol{q}_{n}, \boldsymbol{q}_{n+1}\right) \\
& \quad-\Delta t\left(D_{1} \boldsymbol{\pi}\left(\boldsymbol{q}_{n+\frac{1}{2}}, \boldsymbol{d}_{n+\frac{1}{2}}, \boldsymbol{f}_{n}\right)\right)^{T} \overline{\bar{\nabla}}_{\boldsymbol{\pi}} \boldsymbol{\Psi}\left(\boldsymbol{\pi}_{n}, \boldsymbol{\pi}_{n+1}\right) \cdot \boldsymbol{\lambda}_{n+1} \\
& \mathbf{0}=\left(D_{2} \boldsymbol{\pi}\left(\boldsymbol{q}_{n+\frac{1}{2}}, \boldsymbol{d}_{n+\frac{1}{2}}, \boldsymbol{f}_{n}\right)\right)^{T} \overline{\bar{\nabla}}_{\pi} \boldsymbol{\Psi}\left(\boldsymbol{\pi}_{n}, \boldsymbol{\pi}_{n+1}\right) \cdot \lambda_{n+1} \\
& \mathbf{0}=\boldsymbol{\Psi}\left(\boldsymbol{\pi}\left(\boldsymbol{q}_{n+1}, \boldsymbol{d}_{n+1}, \boldsymbol{f}_{n}\right)\right)
\end{aligned}
$$

Here, the constraints are assembled as follows

$$
\boldsymbol{\Psi}\left(\boldsymbol{\pi}\left(\boldsymbol{q}_{n+1}, \boldsymbol{d}_{n+1}, \boldsymbol{f}_{n}\right)\right)=\left[\begin{array}{l}
\boldsymbol{\Psi}_{\text {mortar }}\left(\boldsymbol{\pi}\left(\boldsymbol{q}_{n+1}, \boldsymbol{d}_{n+1}, \boldsymbol{f}_{n}\right)\right) \\
\boldsymbol{\Psi}_{\text {normal }}\left(\boldsymbol{\pi}\left(\boldsymbol{q}_{n+1}, \boldsymbol{d}_{n+1}, \boldsymbol{f}_{n}\right)\right)
\end{array}\right]
$$

Note that the segments have to be generated merely once for each time step and are held constant until the next time step.

3. A further decrease of the size and the complexity of the system can be achieved by eliminating the augmentation of the normal vector and sacrificing exact conservation of energy. In particular, we retain the augmented coordinates $\boldsymbol{f}_{n}$ and make use of the cubic invariants $\breve{\boldsymbol{\pi}}$ (see (43)) instead of the quadratic invarinats

$$
\begin{aligned}
& \boldsymbol{q}_{n+1}-\boldsymbol{q}_{n}=\Delta t \boldsymbol{M}^{-1} \boldsymbol{p}_{n+\frac{1}{2}} \\
& \boldsymbol{p}_{n+1}-\boldsymbol{p}_{n}=-\Delta t \bar{\nabla} V\left(\boldsymbol{q}_{n}, \boldsymbol{q}_{n+1}\right) \\
& \quad-\Delta t\left(D_{1} \breve{\boldsymbol{\pi}}\left(\boldsymbol{q}_{n+\frac{1}{2}}, \boldsymbol{f}_{n}\right)\right)^{T} \nabla_{\mathrm{M}} \boldsymbol{\Psi}\left(\breve{\boldsymbol{\pi}}_{n}, \breve{\boldsymbol{\pi}}_{n+1}\right) \cdot \boldsymbol{\lambda}_{n+1} \\
& \mathbf{0}=\breve{\boldsymbol{\Psi}}_{\text {mortar }}\left(\breve{\boldsymbol{\pi}}\left(\boldsymbol{q}_{n+1}, \boldsymbol{f}_{n}\right)\right)
\end{aligned}
$$

Analogues to the time-continuous case we can identify several constants of motion in the discrete setting. Again we focus on the constraint contributions and start with the conservation properties of the momentum maps for the first approach

$$
\begin{aligned}
& G\left(z_{n+1}\right)-G\left(z_{n}\right)=\boldsymbol{\mu} \cdot \sum_{A \in \bar{\omega}}\left(\boldsymbol{p}_{A, n+1}-\boldsymbol{p}_{A, n}\right) \\
& =\boldsymbol{\mu} \cdot \sum_{A \in \bar{\omega}}\left(\partial_{\boldsymbol{q}_{A}} \boldsymbol{\pi}\left(\boldsymbol{q}_{n+\frac{1}{2}}, \boldsymbol{d}_{n+\frac{1}{2}}, \boldsymbol{f}_{n+\frac{1}{2}}\right)\right)^{T} \overline{\bar{\nabla}}_{\pi} \boldsymbol{\Psi} \\
& \quad \times\left(\boldsymbol{\pi}_{n}, \boldsymbol{\pi}_{n+1}\right) \cdot \boldsymbol{\lambda}_{n+1}=0
\end{aligned}
$$

and

$$
\begin{aligned}
G\left(z_{n+1}\right)-G\left(z_{n}\right)=\boldsymbol{\mu} \cdot \sum_{A \in \omega}\left[\left(\boldsymbol{q}_{A, n+1}-\boldsymbol{q}_{A, n}\right)\right. \\
\left.\quad \times \boldsymbol{p}_{A, n+\frac{1}{2}}+\boldsymbol{q}_{A, n+\frac{1}{2}} \times\left(\boldsymbol{p}_{n+1}-\boldsymbol{p}_{n}\right)\right] \\
=-\Delta t \boldsymbol{\mu} \cdot \sum_{A \in \omega}\left[\boldsymbol{q}_{A, n+\frac{1}{2}}\right. \\
\left.\quad \times\left(\partial_{\boldsymbol{q}_{A}} \boldsymbol{\pi}\left(\boldsymbol{q}_{n+\frac{1}{2}}, \boldsymbol{d}_{n+\frac{1}{2}}, \boldsymbol{f}_{n+\frac{1}{2}}\right)\right)^{T} \overline{\bar{\nabla}}_{\pi} \boldsymbol{\Psi}\left(\boldsymbol{\pi}_{n}, \boldsymbol{\pi}_{n+1}\right) \cdot \boldsymbol{\lambda}_{n+1}\right] \\
=-\Delta t \boldsymbol{\lambda}_{n+1} \cdot\left(\overline{\bar{\nabla}}_{\pi} \boldsymbol{\Psi}\left(\boldsymbol{\pi}_{n}, \boldsymbol{\pi}_{n+1}\right)\right)^{T} \\
\quad \cdot \sum_{A \in \omega}\left(\partial_{\boldsymbol{q}_{A}} \boldsymbol{\pi}\left(\boldsymbol{q}_{n+\frac{1}{2}}, \boldsymbol{d}_{n+\frac{1}{2}}, \boldsymbol{f}_{n+\frac{1}{2}}\right)\right) \\
\quad \hat{\boldsymbol{q}}_{A, n+\frac{1}{2}} \boldsymbol{\mu}
\end{aligned}
$$

With regard to (56) we can state

$$
\begin{aligned}
& \left(\overline{\bar{\nabla}}_{\pi} \boldsymbol{\Psi}\left(\boldsymbol{\pi}_{n}, \boldsymbol{\pi}_{n+1}\right)\right)^{T} \cdot\left[\sum_{A \in \bar{\omega}}\left(\partial_{\boldsymbol{q}_{A}} \boldsymbol{\pi}\left(\boldsymbol{q}_{n+\frac{1}{2}}, \boldsymbol{d}_{n+\frac{1}{2}}, \boldsymbol{f}_{n+\frac{1}{2}}\right)\right) \cdot \hat{\boldsymbol{q}}_{A, n+\frac{1}{2}} \boldsymbol{\mu}\right. \\
& \left.-\sum_{B \in \omega_{\mathrm{seg}}}\left(\partial_{\boldsymbol{d}_{B}} \boldsymbol{\pi}\left(\boldsymbol{q}_{n+\frac{1}{2}}, \boldsymbol{d}_{n+\frac{1}{2}}, \boldsymbol{f}_{n+\frac{1}{2}}\right)\right) \cdot \hat{\boldsymbol{d}}_{B, n+\frac{1}{2}} \boldsymbol{\mu}\right]=\mathbf{0}
\end{aligned}
$$

and rewrite the last equation

$$
\begin{aligned}
G\left(z_{n+1}\right)-G\left(z_{n}\right) \\
=\Delta t \boldsymbol{\lambda}_{n+1} \cdot\left(\overline{\bar{\nabla}}_{\pi} \boldsymbol{\Psi}\left(\boldsymbol{\pi}_{n}, \boldsymbol{\pi}_{n+1}\right)\right)^{T} \\
\quad \cdot \sum_{B \in \omega_{\mathrm{seg}}}\left[\left(\partial_{\boldsymbol{d}_{B}} \boldsymbol{\pi}\left(\boldsymbol{q}_{n+\frac{1}{2}}, \boldsymbol{d}_{n+\frac{1}{2}}, \boldsymbol{f}_{n+\frac{1}{2}}\right)\right) \cdot \hat{\boldsymbol{d}}_{B, n+\frac{1}{2}} \boldsymbol{\mu}\right] \\
=\Delta t \boldsymbol{\mu} \cdot \sum_{B \in \omega_{\mathrm{seg}}} \boldsymbol{d}_{B, n+\frac{1}{2}} \\
\quad \times\left(\partial_{\boldsymbol{d}_{B}} \boldsymbol{\pi}\left(\boldsymbol{q}_{n+\frac{1}{2}}, \boldsymbol{d}_{n+\frac{1}{2}}, \boldsymbol{f}_{n+\frac{1}{2}}\right)\right)^{T} \overline{\bar{\nabla}}_{\boldsymbol{\pi}} \boldsymbol{\Psi}\left(\boldsymbol{\pi}_{n}, \boldsymbol{\pi}_{n+1}\right) \cdot \boldsymbol{\lambda}_{n+1}=0
\end{aligned}
$$

Next, we verify algorithmic conservation of energy. Since the original system deals with inequality constraints, an additional error in energy arises due to the application of the active set strategy. As shown in Hesch and Betsch [? ], this error is negligible and can be treated as described in the afore mentioned paper. For the algorithmic conservation of energy the net power input to the system within each time step has to be zero. After a few calculations we receive

$$
\begin{aligned}
\Delta E= & \lambda_{n+1} \cdot D_{1} \boldsymbol{\pi}\left(\boldsymbol{q}_{n+\frac{1}{2}}, \boldsymbol{d}_{n+\frac{1}{2}}, \boldsymbol{f}_{n+\frac{1}{2}}\right)^{T} \overline{\bar{\nabla}}_{\pi} \boldsymbol{\Psi}\left(\boldsymbol{\pi}_{n}, \boldsymbol{\pi}_{n+1}\right) \\
& \cdot\left(\boldsymbol{q}_{n+1}-\boldsymbol{q}_{n}\right)
\end{aligned}
$$


where $\Delta E$ denotes the change in total energy. Introducing the discrete consistency condition

$$
\begin{aligned}
D_{1} & \boldsymbol{\pi}\left(\boldsymbol{q}_{n+\frac{1}{2}}, \boldsymbol{d}_{n+\frac{1}{2}}, \boldsymbol{f}_{n+\frac{1}{2}}\right)^{T} \overline{\bar{\nabla}}_{\pi} \boldsymbol{\Psi}\left(\boldsymbol{\pi}_{n}, \boldsymbol{\pi}_{n+1}\right)\left(\boldsymbol{q}_{n+1}-\boldsymbol{q}_{n}\right) \\
& +D_{2} \boldsymbol{\pi}\left(\boldsymbol{q}_{n+\frac{1}{2}}, \boldsymbol{d}_{n+\frac{1}{2}}, \boldsymbol{f}_{n+\frac{1}{2}}\right)^{T} \overline{\bar{\nabla}}_{\pi} \boldsymbol{\Psi}\left(\boldsymbol{\pi}_{n}, \boldsymbol{\pi}_{n+1}\right)\left(\boldsymbol{d}_{n+1}-\boldsymbol{d}_{n}\right) \\
& +D_{3} \boldsymbol{\pi}\left(\boldsymbol{q}_{n+\frac{1}{2}}, \boldsymbol{d}_{n+\frac{1}{2}}, \boldsymbol{f}_{n+\frac{1}{2}}\right)^{T} \overline{\bar{\nabla}}_{\pi} \boldsymbol{\Psi}\left(\boldsymbol{\pi}_{n}, \boldsymbol{\pi}_{n+1}\right)\left(\boldsymbol{f}_{n+1}-\boldsymbol{f}_{n}\right) \\
= & \overline{\bar{\nabla}}_{\pi} \boldsymbol{\Psi}\left(\boldsymbol{\pi}_{n}, \boldsymbol{\pi}_{n+1}\right)\left(\boldsymbol{\pi}_{n+1}-\boldsymbol{\pi}_{n}\right) \\
= & \boldsymbol{\Psi}\left(\boldsymbol{\pi}_{n+1}\right)-\boldsymbol{\Psi}\left(\boldsymbol{\pi}_{n}\right)=\mathbf{0}
\end{aligned}
$$

we can show that

$$
\begin{aligned}
\Delta E= & -\boldsymbol{\lambda}_{n+1} \cdot\left[D_{2} \boldsymbol{\pi}\left(\boldsymbol{q}_{n+\frac{1}{2}}, \boldsymbol{d}_{n+\frac{1}{2}}, \boldsymbol{f}_{n+\frac{1}{2}}\right)^{T}\right. \\
& \times \overline{\bar{\nabla}}_{\pi} \boldsymbol{\Psi}\left(\boldsymbol{\pi}_{n}, \boldsymbol{\pi}_{n+1}\right)\left(\boldsymbol{d}_{n+1}-\boldsymbol{d}_{n}\right) \\
& +D_{3} \boldsymbol{\pi}\left(\boldsymbol{q}_{n+\frac{1}{2}}, \boldsymbol{d}_{n+\frac{1}{2}}, \boldsymbol{f}_{n+\frac{1}{2}}\right)^{T} \\
& \left.\times \overline{\bar{\nabla}}_{\pi} \boldsymbol{\Psi}\left(\boldsymbol{\pi}_{n}, \boldsymbol{\pi}_{n+1}\right)\left(\boldsymbol{f}_{n+1}-\boldsymbol{f}_{n}\right)\right] \\
= & 0
\end{aligned}
$$

is valid. Thus, total energy is conserved.

Similarly, the second approach yields for the momentum maps

$$
\begin{aligned}
& G\left(z_{n+1}\right)-G\left(z_{n}\right)=\boldsymbol{\mu} \cdot \sum_{A \in \bar{\omega}}\left(\boldsymbol{p}_{A, n+1}-\boldsymbol{p}_{A, n}\right) \\
& =\boldsymbol{\mu} \cdot \sum_{A \in \bar{\omega}}\left(\partial_{\boldsymbol{q}_{A}} \boldsymbol{\pi}\left(\boldsymbol{q}_{n+\frac{1}{2}}, \boldsymbol{d}_{n+\frac{1}{2}}, \boldsymbol{f}_{n}\right)\right)^{T} \\
& \quad \times \overline{\bar{\nabla}}_{\pi} \boldsymbol{\Psi}\left(\boldsymbol{\pi}_{n}, \boldsymbol{\pi}_{n+1}\right) \cdot \boldsymbol{\lambda}_{n+1} \\
& =0
\end{aligned}
$$

and

$$
\begin{aligned}
& G\left(z_{n+1}\right)-G\left(z_{n}\right)=\boldsymbol{\mu} \cdot \sum_{A \in \omega}\left[\left(\boldsymbol{q}_{A, n+1}-\boldsymbol{q}_{A, n}\right) \times \boldsymbol{p}_{A, n+\frac{1}{2}}\right. \\
& \left.+\boldsymbol{q}_{A, n+\frac{1}{2}} \times\left(\boldsymbol{p}_{n+1}-\boldsymbol{p}_{n}\right)\right] \\
& =-\Delta t \boldsymbol{\mu} \cdot \sum_{A \in \omega}\left[\boldsymbol{q}_{A, n+\frac{1}{2}}\right. \\
& \left.\times\left(\partial_{\boldsymbol{q}_{A}} \boldsymbol{\pi}\left(\boldsymbol{q}_{n+\frac{1}{2}}, \boldsymbol{d}_{n+\frac{1}{2}}, \boldsymbol{f}_{n}\right)\right)^{T} \overline{\bar{\nabla}}_{\pi} \boldsymbol{\Psi}\left(\boldsymbol{\pi}_{n}, \boldsymbol{\pi}_{n+1}\right) \cdot \boldsymbol{\lambda}_{n+1}\right] \\
& =-\Delta t \lambda_{n+1} \cdot\left(\overline{\bar{\nabla}}_{\pi} \boldsymbol{\Psi}\left(\boldsymbol{\pi}_{n}, \boldsymbol{\pi}_{n+1}\right)\right)^{T} \\
& \cdot \sum_{A \in \omega}\left(\partial_{\boldsymbol{q}_{A}} \boldsymbol{\pi}\left(\boldsymbol{q}_{n+\frac{1}{2}}, \boldsymbol{d}_{n+\frac{1}{2}}, \boldsymbol{f}_{n}\right)\right) \cdot \hat{\boldsymbol{q}}_{A, n+\frac{1}{2}} \boldsymbol{\mu}
\end{aligned}
$$

With regard to (56) we obtain immediately

$$
\begin{aligned}
& \left(\overline{\bar{\nabla}} \bar{\pi} \boldsymbol{\Psi}\left(\boldsymbol{\pi}_{n}, \boldsymbol{\pi}_{n+1}\right)\right)^{T} \\
& \quad\left[\sum_{A \in \bar{\omega}}\left(\partial_{\boldsymbol{q}_{A}} \boldsymbol{\pi}\left(\boldsymbol{q}_{n+\frac{1}{2}}, \boldsymbol{d}_{n+\frac{1}{2}}, \boldsymbol{f}_{n}\right)\right) \cdot \hat{\boldsymbol{q}}_{A, n+\frac{1}{2}} \boldsymbol{\mu}\right. \\
& \left.\quad-\sum_{B \in \omega_{\mathrm{seg}}}\left(\partial_{\boldsymbol{d}_{B}} \boldsymbol{\pi}\left(\boldsymbol{q}_{n+\frac{1}{2}}, \boldsymbol{d}_{n+\frac{1}{2}}, \boldsymbol{f}_{n}\right)\right) \cdot \hat{\boldsymbol{d}}_{B, n+\frac{1}{2}} \boldsymbol{\mu}\right]=\mathbf{0}
\end{aligned}
$$

and rewrite Eq. (73) as follows

$$
\begin{aligned}
G\left(z_{n+1}\right)-G\left(z_{n}\right) & \\
= & \Delta t \boldsymbol{\lambda}_{n+1} \cdot\left(\overline{\bar{\nabla}}_{\pi} \boldsymbol{\Psi}\left(\boldsymbol{\pi}_{n}, \boldsymbol{\pi}_{n+1}\right)\right)^{T} \\
& \cdot \sum_{B \in \omega_{\mathrm{seg}}}\left[\left(\partial_{\boldsymbol{d}_{B}} \boldsymbol{\pi}\left(\boldsymbol{q}_{n+\frac{1}{2}}, \boldsymbol{d}_{n+\frac{1}{2}}, \boldsymbol{f}_{n}\right)\right) \cdot \hat{\boldsymbol{d}}_{B, n+\frac{1}{2}} \boldsymbol{\mu}\right] \\
= & \Delta t \boldsymbol{\mu} \cdot \sum_{B \in \omega_{\mathrm{seg}}} \boldsymbol{d}_{B, n+\frac{1}{2}} \\
& \times\left(\partial_{\boldsymbol{d}_{B}} \boldsymbol{\pi}\left(\boldsymbol{q}_{n+\frac{1}{2}}, \boldsymbol{d}_{n+\frac{1}{2}}, \boldsymbol{f}_{n}\right)\right)^{T} \overline{\bar{\nabla}}_{\pi} \boldsymbol{\Psi}\left(\boldsymbol{\pi}_{n}, \boldsymbol{\pi}_{n+1}\right) \cdot \boldsymbol{\lambda}_{n+1} \\
= & 0
\end{aligned}
$$

Again, both momentum maps are algorithmically conserved. For the total energy we get

$$
\begin{aligned}
\Delta E= & \lambda_{n+1} \cdot D_{1} \boldsymbol{\pi}\left(\boldsymbol{q}_{n+\frac{1}{2}}, \boldsymbol{d}_{n+\frac{1}{2}}, \boldsymbol{f}_{n}\right)^{T} \overline{\bar{\nabla}}_{\pi} \boldsymbol{\Psi}\left(\boldsymbol{\pi}_{n}, \boldsymbol{\pi}_{n+1}\right) \\
& \cdot\left(\boldsymbol{q}_{n+1}-\boldsymbol{q}_{n}\right)
\end{aligned}
$$

The discrete consistency condition reads

$$
\begin{aligned}
D_{1} & \boldsymbol{\pi}\left(\boldsymbol{q}_{n+\frac{1}{2}}, \boldsymbol{d}_{n+\frac{1}{2}}, \boldsymbol{f}_{n}\right)^{T} \overline{\bar{\nabla}}_{\pi} \boldsymbol{\Psi}\left(\boldsymbol{\pi}_{n}, \boldsymbol{\pi}_{n+1}\right)\left(\boldsymbol{q}_{n+1}-\boldsymbol{q}_{n}\right) \\
& \quad+D_{2} \boldsymbol{\pi}\left(\boldsymbol{q}_{n+\frac{1}{2}}, \boldsymbol{d}_{n+\frac{1}{2}}, \boldsymbol{f}_{n}\right)^{T} \overline{\bar{\nabla}}_{\pi} \boldsymbol{\Psi}\left(\boldsymbol{\pi}_{n}, \boldsymbol{\pi}_{n+1}\right)\left(\boldsymbol{d}_{n+1}-\boldsymbol{d}_{n}\right) \\
= & \overline{\bar{\nabla}}_{\pi} \boldsymbol{\Psi}\left(\boldsymbol{\pi}_{n}, \boldsymbol{\pi}_{n+1}\right)\left(\boldsymbol{\pi}_{n+1}-\boldsymbol{\pi}_{n}\right) \\
= & \boldsymbol{\Psi}\left(\boldsymbol{\pi}_{n+1}\right)-\boldsymbol{\Psi}\left(\boldsymbol{\pi}_{n}\right)=\mathbf{0}
\end{aligned}
$$

and insertion in (76) yields

$$
\begin{aligned}
\Delta E= & -\boldsymbol{\lambda}_{n+1} \cdot\left[D_{2} \boldsymbol{\pi}\left(\boldsymbol{q}_{n+\frac{1}{2}}, \boldsymbol{d}_{n+\frac{1}{2}}, \boldsymbol{f}_{n}\right)^{T}\right. \\
& \left.\times \overline{\bar{\nabla}}_{\pi} \boldsymbol{\Psi}\left(\boldsymbol{\pi}_{n}, \boldsymbol{\pi}_{n+1}\right)\left(\boldsymbol{d}_{n+1}-\boldsymbol{d}_{n}\right)\right]=0
\end{aligned}
$$

Thus, total energy is conserved.

At last, we can show for the third approach that

$$
\begin{aligned}
& G\left(z_{n+1}\right)-G\left(z_{n}\right)=\boldsymbol{\mu} \cdot \sum_{A \in \bar{\omega}}\left(\boldsymbol{p}_{A, n+1}-\boldsymbol{p}_{A, n}\right) \\
& \quad=\boldsymbol{\mu} \cdot \sum_{A \in \bar{\omega}}\left(\partial_{\boldsymbol{q}_{A}} \breve{\boldsymbol{\pi}}\left(\boldsymbol{q}_{n+\frac{1}{2}}, \boldsymbol{f}_{n}\right)\right)^{T} \nabla_{\breve{\pi}} \breve{\boldsymbol{\Psi}}\left(\breve{\boldsymbol{\pi}}_{n}, \breve{\boldsymbol{\pi}}_{n+1}\right) \cdot \lambda_{n+1} \\
& \quad=0
\end{aligned}
$$

and

$$
\begin{aligned}
& G\left(z_{n+1}\right)-G\left(z_{n}\right)=\boldsymbol{\mu} \cdot \sum_{A \in \omega}\left[\left(\boldsymbol{q}_{A, n+1}-\boldsymbol{q}_{A, n}\right) \times \boldsymbol{p}_{A, n+\frac{1}{2}}\right. \\
& \left.+\boldsymbol{q}_{A, n+\frac{1}{2}} \times\left(\boldsymbol{p}_{n+1}-\boldsymbol{p}_{n}\right)\right] \\
& =-\Delta t \boldsymbol{\mu} \cdot \sum_{A \in \omega}\left[\boldsymbol{q}_{A, n+\frac{1}{2}}\right.
\end{aligned}
$$

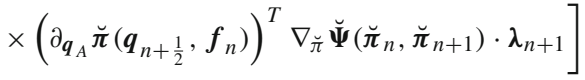

$$
\begin{aligned}
& =-\Delta t \lambda_{n+1} \cdot\left(\nabla_{\breve{\pi}} \breve{\Psi}\left(\breve{\pi}_{n}, \breve{\pi}_{n+1}\right)\right)^{T} \\
& \cdot \sum_{A \in \omega}\left(\partial_{\boldsymbol{q}_{A}} \breve{\pi}\left(\boldsymbol{q}_{n+\frac{1}{2}}, \boldsymbol{f}_{n}\right)\right) \cdot \hat{\boldsymbol{q}}_{A, n+\frac{1}{2}} \boldsymbol{\mu} \\
& =0
\end{aligned}
$$


Fig. 5 Configurations at time $t=2$ and $t=5$

Fig. 6 Segmentation at time $t=2$ and $t=5$
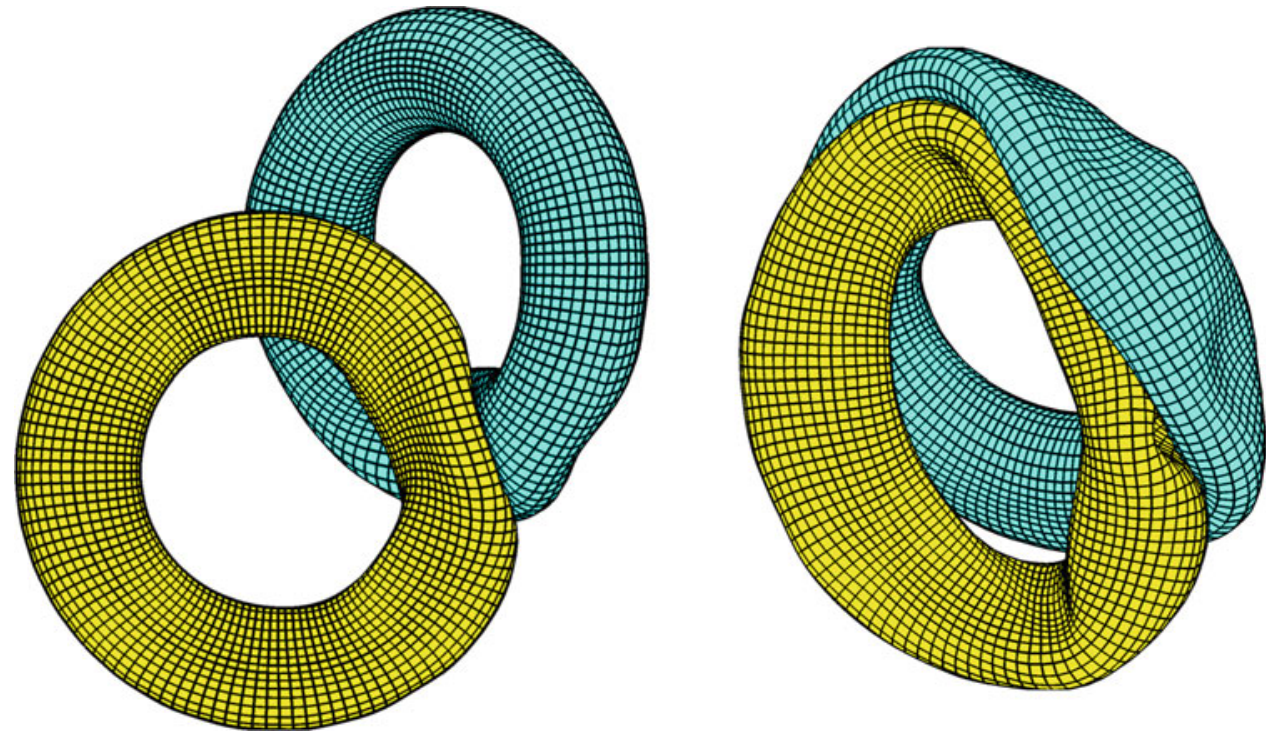

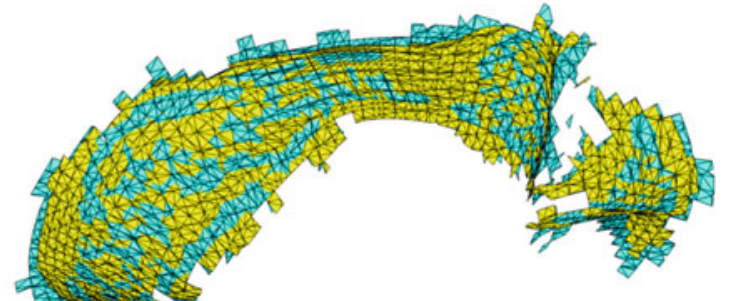

Accordingly, both momentum maps are algorithmically conserved. Since we can not apply the concept of a discrete gradient, total energy is not conserved.

\section{Numerical investigations}

In this section we investigate the performance of the different proposed approaches. Two model problems have been taken from Yang and Lausen [36] and applied to the newly developed schemes.

\subsection{Two tori impact problem}

As a first example we consider an impact simulation of two tori. Both tori are discretized using 8,024 eight-node brick elements with overall 72,216 degrees of freedom. The inner and outer radii are 52 and 100, respectively, the wall thickness of each hollow torus is 4.5. A standard Neo-Hookean hyperelastic material with $\mathcal{E}=2,250$ and $v=0.3$ is used. The initial density $\rho=0.1$ and the homogeneous, initial velocity of the left torus is given

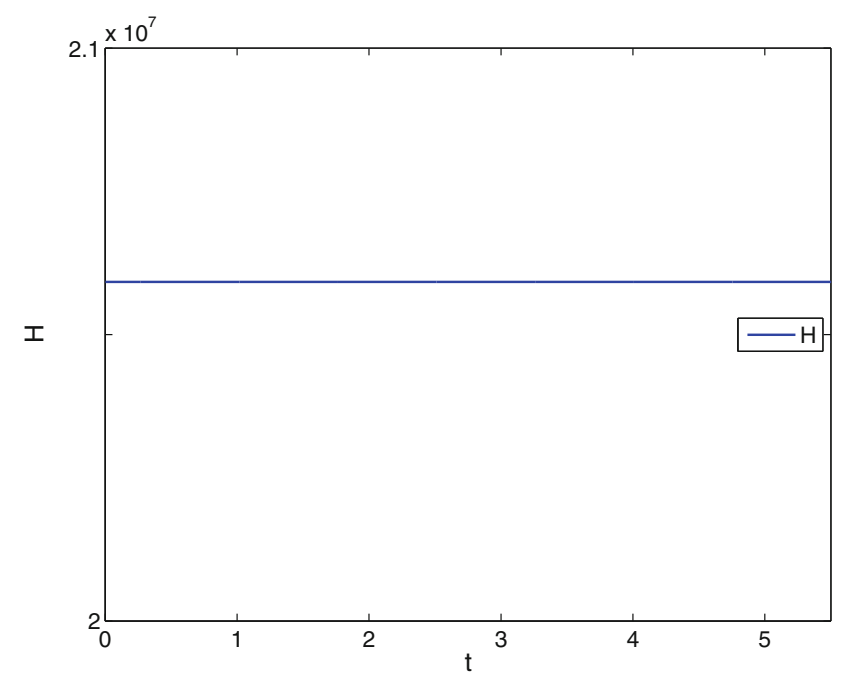

Fig. 7 Total energy versus time using approach 1

by $\boldsymbol{v}=[30,0,23]$. A time-step size of $\Delta t=0.0025$ has been used for the first approach, whereas a timestep size of both simplified approaches has been set to $\Delta t=0.01$. 


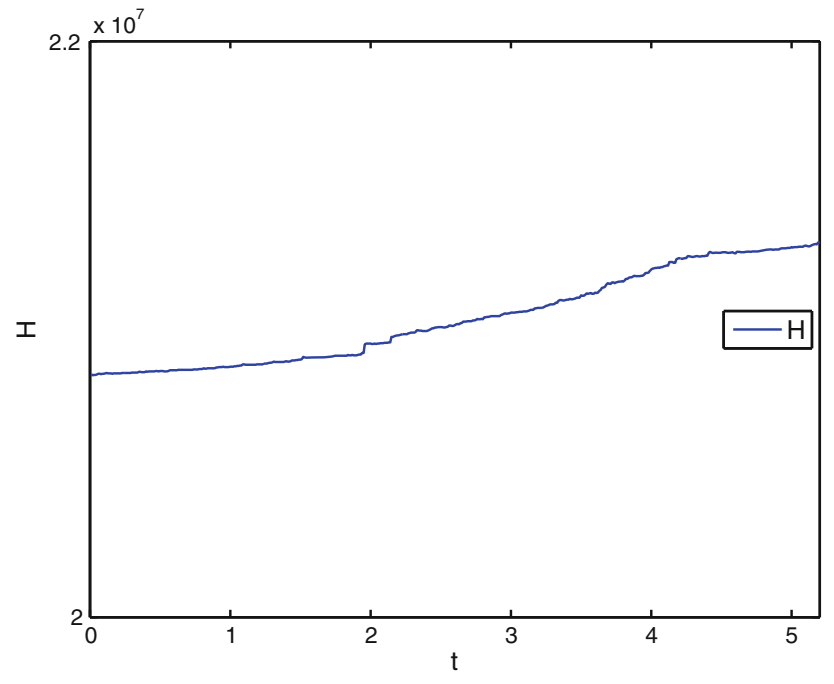

Fig. 8 Total energy versus time using approach 3

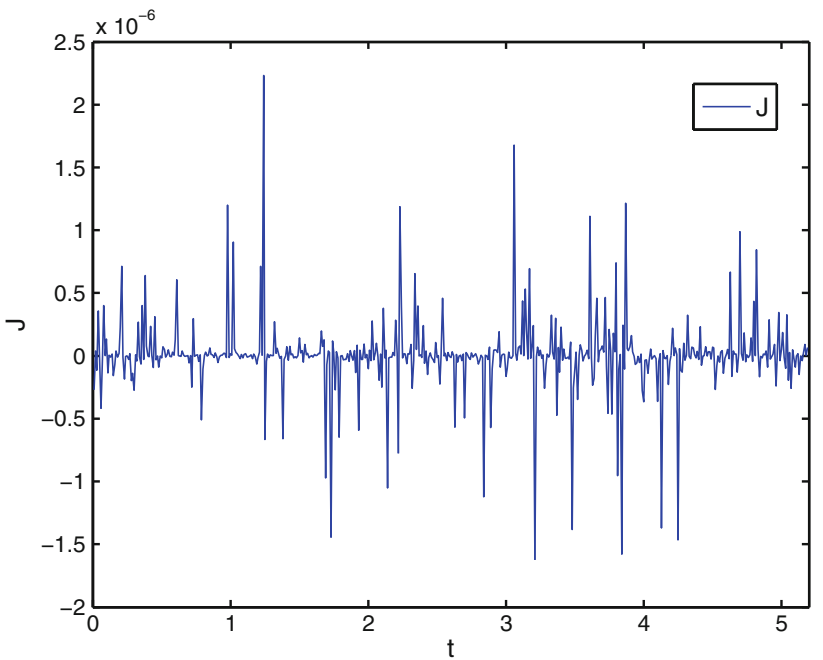

Fig. 9 Values of change of the first component of angular momentum versus time using approach 3

Excluding the augmented coordinates $\boldsymbol{f}$ from the calculations as shown in (62), a reduction of the average calculation time of a Newton step of about $12 \%$ could be achieved in this specific example. Furthermore, we were able to use four to ten times larger time-step sizes. This stability feature is of major importance especially in large sliding situations.

In Fig. 5 the configuration at $t=2$ and $t=5$ is shown. The associated segmentations are displayed in Fig. 6. 2,318 segments are automatically constructed at time $t=2$ for overall 331 mortar constraints. For the first approach where the mortar integrals remain not constant throughout each time step, an additional 355 constraints for $\boldsymbol{\Psi}_{\mathrm{Aug}_{1}}, 240$ constraints for $\boldsymbol{\Psi}_{\mathrm{Aug}_{2}}$ and 703 constraints for $\boldsymbol{\Psi}_{\mathrm{Aug}_{3}}$ are necessary. Furthermore, 6,954 constraints for the augmentation of the normal vector have to be considered. At $t=55,896$ segments with overall 755 mortar constraints are determined. Correspondingly, we need 923 constraints for $\boldsymbol{\Psi}_{\mathrm{Aug}_{1}}, 529$ constraints for $\boldsymbol{\Psi}_{\mathrm{Aug}_{2}}, 1,866$ constraints for $\boldsymbol{\Psi}_{\mathrm{Aug}_{3}}$ and 20,862 constraints for the augmentation of the normal vector. Clearly, this is not acceptable. In contrast, if we apply approach 3 , we only have to add 755 mortar constraints to the global system. We then need the same amount of constraints as for the NTS method, since each mortar constraint refers to a specific node on the non-mortar side. Although the evaluation of the mortar constraints is more involved, the solver clearly dominates the overall calculation time and thus, we have no drawback in the calculation time due to the use of mortar methods.

In Fig. 7 total energy versus time is displayed using the proposed energy-momentum scheme together with deformable mortar segments. As shown in Fig. 8, approach 3 does not conserve energy. The increase in total energy is acceptable, since we used relatively large time steps. The last diagram shows the values of change of the first component of angular momentum. Note that the values are below the stopping criterion of the Newton iteration $\left(10^{-5}\right)$ (Fig. 9).

\subsection{Torus-cylinder impact example}

As before, we utilize a problem introduced by Yang and Laursen [36], see Fig. 10. The material properties and the initial geometry of the torus are the same as in Sect. 6.1. The inner diameter of the cylinder is 100 , the wall thickness is 7.5 and
Fig. 10 Configuration at time $t=0$ and $t=5.8$
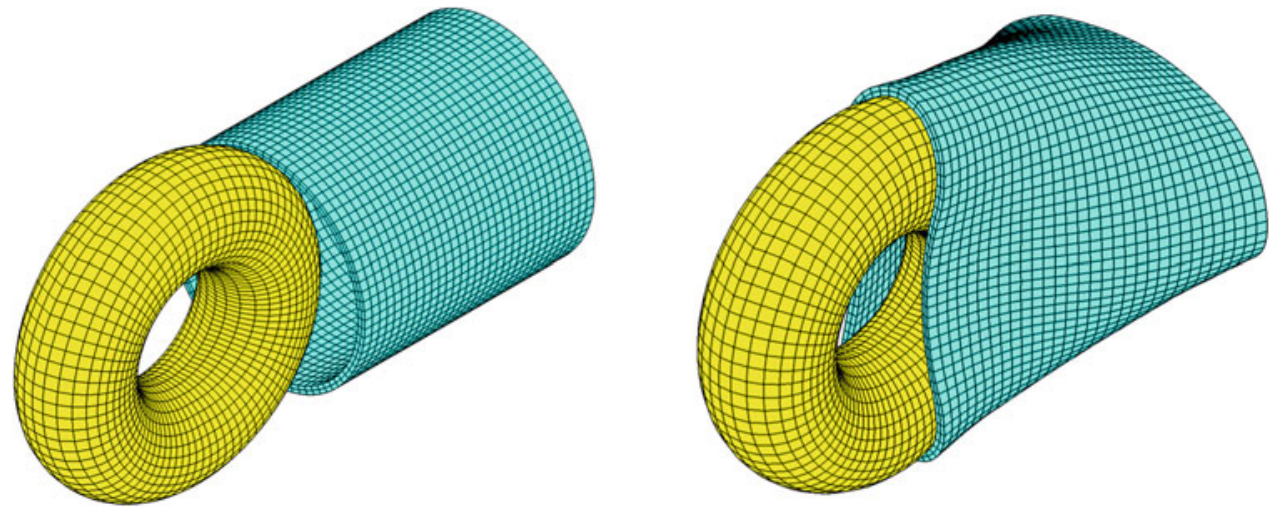


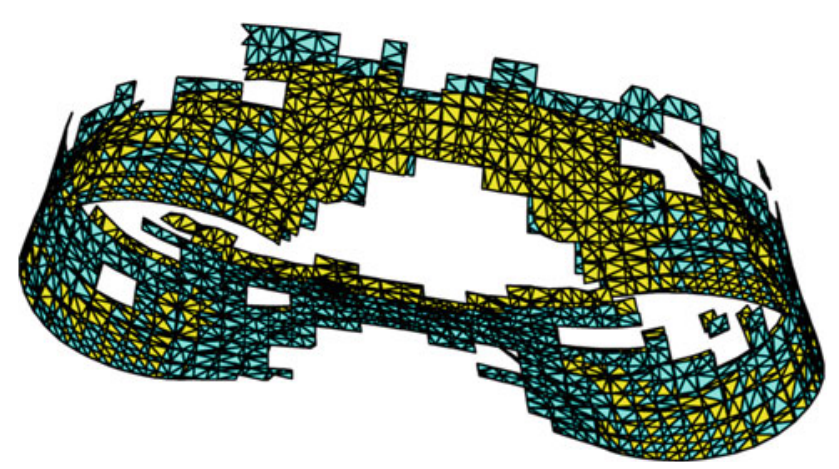

Fig. 11 Segmentation at time $t=5.8\left(\right.$ rotated $\left.90^{\circ}\right)$

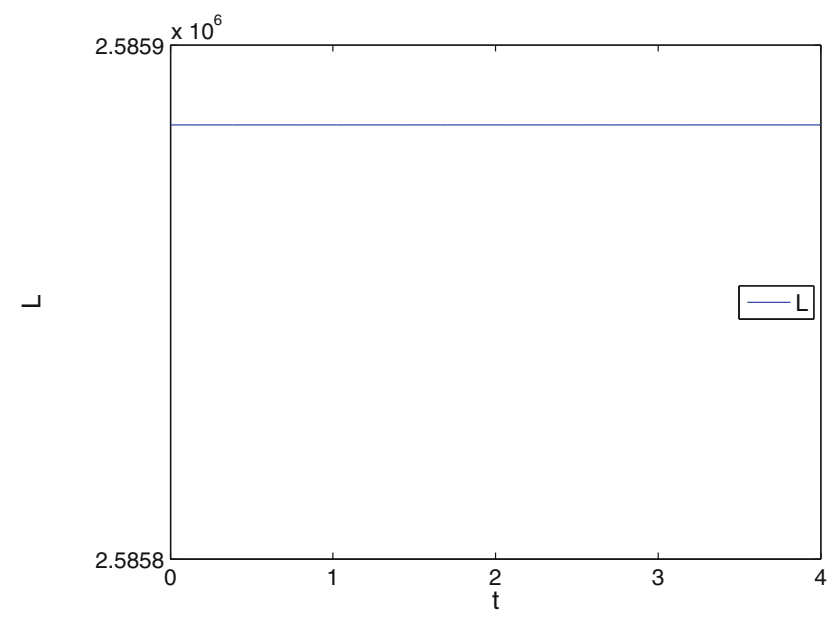

Fig. 12 Linear momentum versus time

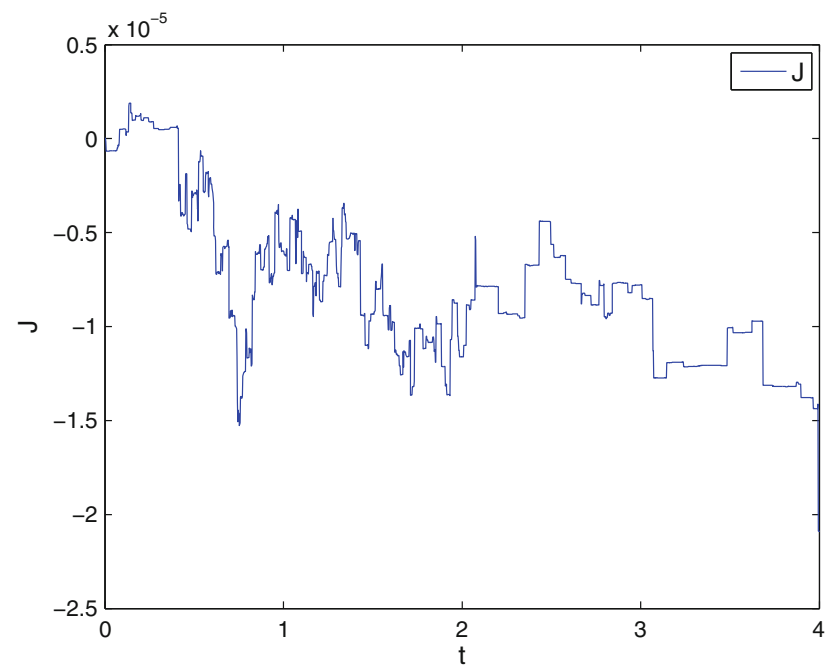

Fig. 13 Third component of the angular momentum versus time

the initial velocity of the torus is $[0,0,20]$. The Torus consists of 4,992 elements and 22,464 degrees of freedom, the cylinder of 4,960 elements and 23,040 degrees of freedom. Furthermore, the time-step size has been set to $\Delta t=0.005$. A standard Neo-Hookean hyperelastic material with $\mathcal{E}=$
12000 and $v=0.3$ is used. In Fig. 11 a typical segmentation after $5.8 \mathrm{~s}$ is displayed.

As before, the vector of augmented coordinates would become unacceptably large. Thus, we concentrate on approach 3. As can be seen in Figs. 12 and 13, respectively, linear and angular momentum are algorithmically conserved.

\section{Conclusions}

This paper extends the mixed energy momentum approach, developed previously in the context of the NTS method, to the mortar method. The proposed methods conserve linear and angular momentum algorithmically, which is new in the context of mortar contact methods. To achieve this benefit, an accurate segmentation procedure as well as the reformulation of the constraints in terms of invariants using reducible coordinates is presented in detail. Furthermore, an energy conserving algorithm has been applied to the constraints.

We have also shown how to apply several simplifications without affecting conservation of the momentum maps. These simplifications lead to a reduction of the numerical costs of the mortar method to a level, similar to the costs of the traditional NTS method. This comes at the expense of algorithmic energy conservation.

Acknowledgments Support for this research was provided by the Deutsche Forschungsgemeinschaft (DFG) under grant HE 5943/1-1. This support is gratefully acknowledged.

\section{Appendix A: Reformulation of the additional constraints}

For the reformulation of (16) in terms of invariants similar to (34), we define a vector of possible invariants as follows

$$
\boldsymbol{\pi}=\left[\begin{array}{c}
\left(\boldsymbol{q}_{J}^{(1)}-\boldsymbol{q}_{1}^{(1)}\right) \cdot\left(\boldsymbol{q}_{I}^{(2)}-\boldsymbol{q}_{1}^{(1)}\right) \\
\left(\boldsymbol{q}_{J}^{(1)}-\boldsymbol{q}_{1}^{(1)}\right) \cdot\left(\boldsymbol{q}_{J}^{(1)}-\boldsymbol{q}_{1}^{(1)}\right) \\
\left(\boldsymbol{q}_{2}^{(1)}-\boldsymbol{q}_{1}^{(1)}\right) \cdot\left(\boldsymbol{q}_{3}^{(1)}-\boldsymbol{q}_{1}^{(1)}\right) \\
\left(\boldsymbol{q}_{2}^{(1)}-\boldsymbol{q}_{1}^{(1)}\right) \cdot\left(\boldsymbol{q}_{4}^{(1)}-\boldsymbol{q}_{1}^{(1)}\right) \\
\left(\boldsymbol{q}_{3}^{(1)}-\boldsymbol{q}_{1}^{(1)}\right) \cdot\left(\boldsymbol{q}_{4}^{(1)}-\boldsymbol{q}_{1}^{(1)}\right) \\
\overline{\boldsymbol{\xi}}_{I}^{(1)}
\end{array}\right], \quad J \in[2,3,4]
$$

for all projected nodes $I$. For the reformulated constraints we obtain

$$
\begin{aligned}
& \boldsymbol{\Psi}_{\mathrm{Aug}_{1}, I}(\boldsymbol{\pi}) \\
& =\left[\begin{array}{l}
{\left[N_{2, \pi_{10}} N_{3, \pi_{10}} N_{4, \pi_{10}}\right] \cdot\left\{\left[\begin{array}{l}
\pi_{1} \\
\pi_{2} \\
\pi_{3}
\end{array}\right]-N_{2}\left[\begin{array}{l}
\pi_{4} \\
\pi_{7} \\
\pi_{8}
\end{array}\right]-N_{3}\left[\begin{array}{l}
\pi_{7} \\
\pi_{5} \\
\pi_{9}
\end{array}\right]-N_{4}\left[\begin{array}{l}
\pi_{8} \\
\pi_{9} \\
\pi_{6}
\end{array}\right]\right\}} \\
{\left[N_{2, \pi_{11}} N_{3, \pi_{11}} N_{4, \pi_{11}}\right] \cdot\left\{\left[\begin{array}{l}
\pi_{1} \\
\pi_{2} \\
\pi_{3}
\end{array}\right]-N_{2}\left[\begin{array}{l}
\pi_{4} \\
\pi_{7} \\
\pi_{8}
\end{array}\right]-N_{3}\left[\begin{array}{l}
\pi_{7} \\
\pi_{5} \\
\pi_{9}
\end{array}\right]-N_{4}\left[\begin{array}{l}
\pi_{8} \\
\pi_{9} \\
\pi_{6}
\end{array}\right]\right\}}
\end{array}\right]
\end{aligned}
$$


where $N_{J, \pi_{10}}\left(\pi_{10}, \pi_{11}\right)$ denotes the derivative of the shape function $J$ with respect to $\pi_{10}$. Moreover, we have to reformulate (17). It is easy to show, that (17) can be rewritten as follows

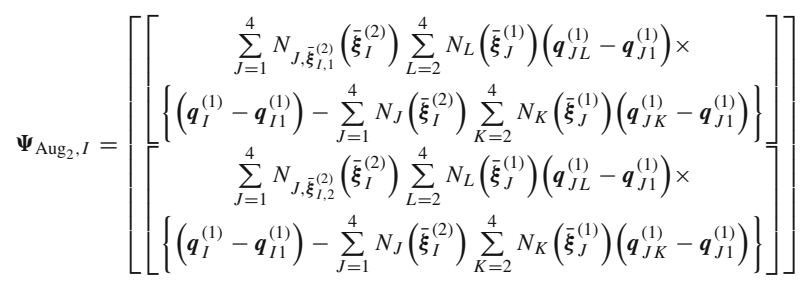

Although tedious, it is straight forward to reformulate Eq. (83) in terms of the invariants

$\boldsymbol{\pi}=\left[\begin{array}{c}\left(\boldsymbol{q}_{J L}^{(1)}-\boldsymbol{q}_{J 1}^{(1)}\right) \cdot\left(\boldsymbol{q}_{J K}^{(1)}-\boldsymbol{q}_{J 1}^{(1)}\right) \\ \overline{\boldsymbol{\xi}}_{J}^{(1)} \\ \overline{\boldsymbol{\xi}}_{I}^{(2)}\end{array}\right]$

Note, that each $\boldsymbol{q}_{J L}^{(1)}, \boldsymbol{q}_{J K}^{(1)}, \forall L, K \in\{2,3,4\}$ depends on the corresponding position of the projection $J \in\{1,2,3,4\}$. Note that we search for the convective coordinates of the mortar side in terms of the projected mesh.

At last we rewrite (41) as follows

$$
\begin{aligned}
& \Psi_{\mathrm{Aug}_{3}}^{\mathrm{ed} I J} \\
& {\left[\begin{array}{c}
{\left[\begin{array}{c}
\sum_{I=1}^{2} \hat{N}_{I}\left(\tilde{\xi}_{1}\right)\left[\sum_{L=1}^{4} N_{L}\left(\bar{\xi}_{I}^{(1)}\right) \boldsymbol{d}_{1}^{(1)} \cdot\left(\boldsymbol{q}_{I L}^{(1)}-\boldsymbol{q}_{1}^{(1)}\right)\right] \\
-\sum_{J=1}^{2} \hat{N}_{J}\left(\tilde{\xi}_{2}\right)\left[\boldsymbol{d}_{1}^{(1)} \cdot\left(\boldsymbol{q}_{J}^{(1)}-\boldsymbol{q}_{1}^{(1)}\right)+\boldsymbol{d}_{1}^{(1)} \cdot \boldsymbol{d}_{J}^{(1)} \tilde{\xi}_{3}\right]
\end{array}\right]} \\
{\left[\begin{array}{c}
\sum_{I=1}^{2} \hat{N}_{I}\left(\tilde{\xi}_{1}\right)\left[\sum_{L=1}^{4} N_{L}\left(\bar{\xi}_{I}^{(1)}\right)\left(\boldsymbol{q}_{1}^{(1)}-\boldsymbol{q}_{2}^{(1)}\right) \cdot\left(\boldsymbol{q}_{I L}^{(1)}-\boldsymbol{q}_{1}^{(1)}\right)\right] \\
-\hat{q}_{J}^{2} \hat{N}_{J}\left(\tilde{\xi}_{2}\right)\left[\left(\boldsymbol{q}_{1}^{(1)}-\boldsymbol{q}_{2}^{(1)}\right) \cdot\left(\boldsymbol{q}_{J}^{(1)}-\boldsymbol{q}_{1}^{(1)}\right)+\left(\boldsymbol{q}_{1}^{(1)}-\boldsymbol{q}_{2}^{(1)}\right) \cdot \boldsymbol{d}_{J}^{(1)} \tilde{\xi}_{3}\right]
\end{array}\right]} \\
\sum_{J=1}^{2} \hat{N}_{I}\left(\tilde{\xi}_{1}\right)\left(\sum_{L=1}^{4} N_{L}\left(\bar{\xi}_{1}^{(1)}\right)\left(\boldsymbol{q}_{1 L}^{(1)}-\boldsymbol{q}_{1}^{(1)}\right)-\right. \\
{\left[\begin{array}{c}
\left.\sum_{K=1}^{4} N_{K}\left(\bar{\xi}_{2}^{(1)}\right)\left(\boldsymbol{q}_{2 K}^{(1)}-\boldsymbol{q}_{1}^{(1)}\right)\right) \cdot\left[\left(\sum_{M=1}^{4} N_{M}\left(\bar{\xi}_{2}^{(1)}\right)\left(\boldsymbol{q}_{2 M}^{(1)}-\boldsymbol{q}_{1}^{(1)}\right)\right)\right] \\
\sum_{J=1}^{2} \hat{N}_{J}\left(\tilde{\xi}_{2}\right)\left(\sum_{L=1}^{4} N_{L}\left(\bar{\xi}_{1}^{(1)}\right)\left(\boldsymbol{q}_{1 L}^{(1)}-\boldsymbol{q}_{1}^{(1)}\right)-\right. \\
\left.\sum_{K=1}^{4} N_{K}\left(\bar{\xi}_{2}^{(1)}\right)\left(\boldsymbol{q}_{2 K}^{(1)}-\boldsymbol{q}_{1}^{(1)}\right)\right) \cdot\left[\left(\boldsymbol{q}_{J}^{(1)}-\boldsymbol{q}_{1}^{(1)}\right)+\boldsymbol{d}_{J}^{(1)} \tilde{\xi}_{3}\right]
\end{array}\right]}
\end{array}\right]}
\end{aligned}
$$

Even more tedious as before, but straight forward we can reformulate the last set of equations in terms of the invariants

$$
\boldsymbol{\pi}=\left[\begin{array}{c}
\boldsymbol{d}_{1}^{(1)} \cdot \boldsymbol{d}_{J}^{(1)} \\
\boldsymbol{d}_{1}^{(1)} \cdot\left(\boldsymbol{q}_{I L}^{(1)}-\boldsymbol{q}_{1}^{(1)}\right) \\
\boldsymbol{d}_{1}^{(1)} \cdot\left(\boldsymbol{q}_{J}^{(1)}-\boldsymbol{q}_{1}^{(1)}\right) \\
\left(\boldsymbol{q}_{1}^{(1)}-\boldsymbol{q}_{2}^{(1)}\right) \cdot \boldsymbol{d}_{J}^{(1)} \\
\left(\boldsymbol{q}_{1 L}^{(1)}-\boldsymbol{q}_{1}^{(1)}\right) \cdot \boldsymbol{d}_{J}^{(1)} \\
\left(\boldsymbol{q}_{2 K}^{(1)}-\boldsymbol{q}_{1}^{(1)}\right) \cdot \boldsymbol{d}_{J}^{(1)} \\
\left(\boldsymbol{q}_{1}^{(1)}-\boldsymbol{q}_{2}^{(1)}\right) \cdot\left(\boldsymbol{q}_{I L}^{(1)}-\boldsymbol{q}_{1}^{(1)}\right) \\
\left(\boldsymbol{q}_{1}^{(1)}-\boldsymbol{q}_{2}^{(1)}\right) \cdot\left(\boldsymbol{q}_{J}^{(1)}-\boldsymbol{q}_{1}^{(1)}\right) \\
\left(\boldsymbol{q}_{1 L}^{(1)}-\boldsymbol{q}_{1}^{(1)}\right) \cdot\left(\boldsymbol{q}_{2 M}^{(1)}-\boldsymbol{q}_{1}^{(1)}\right) \\
\left(\boldsymbol{q}_{2 K}^{(1)}-\boldsymbol{q}_{1}^{(1)}\right) \cdot\left(\boldsymbol{q}_{2 M}^{(1)}-\boldsymbol{q}_{1}^{(1)}\right) \\
\left(\boldsymbol{q}_{1 L}^{(1)}-\boldsymbol{q}_{1}^{(1)}\right) \cdot\left(\boldsymbol{q}_{J}^{(1)}-\boldsymbol{q}_{1}^{(1)}\right) \\
\left(\boldsymbol{q}_{2 K}^{(1)}-\boldsymbol{q}_{1}^{(1)}\right) \cdot\left(\boldsymbol{q}_{J}^{(1)}-\boldsymbol{q}_{1}^{(1)}\right)
\end{array}\right]
$$

together with the convective coordinates $\bar{\xi}_{I}^{(1)}, \tilde{\xi}_{1}, \tilde{\xi}_{2}, \tilde{\xi}_{3}$ for all combinations of $L, M, K \in\{1,2,3,4\}$ and $I, J \in$ $\{1,2,3,4\}$.

\section{References}

1. Armero F, Petöcz E (1998) Formulation and analysis of conserving algorithms for frictionless dynamic contact/impact problems. Comput Methods Appl Mech Eng 158:269-300

2. Bernardi C, Mayday Y, Patera AT (1994) A new nonconforming approach to domain decomposition: the mortar element method. In: Nonlinear partial differential equations and their applications, pp 13-51

3. Betsch P, Steinmann P (2001) Conserving properties of a time FE method-part II: time-stepping schemes for non-linear elastodynamics. Int J Numer Methods Eng 50:1931-1955

4. Betsch P, Steinmann P (2002) Conservation properties of a time FE method. Part III: mechanical systems with holonomic constraints. Int J Numer Methods Eng 53:2271-2304

5. Betsch P, Uhlar S (2007) Energy-momentum conserving integration of multibody dynamics. Multibody Syst Dyn 17(4):243-289

6. Chawla V, Laursen TA (1998) Energy consistent algorithms for frictional contact problems. Int J Numer Methods Eng 42:799-827

7. El-Abbasi N, Bathe KJ (2001) Stability and patch test performance of contact discretizations and a new solution algorithm. Int J Numer Methods Eng 79:1473-1486

8. Fischer KA, Wriggers P (2005) Frictionless $2 d$ contact formulations for finite deformations based on the mortar method. Comput Mech 36:226-244

9. Flemisch B, Puso MA, Wohlmuth BI (2005) A new dual mortar method for curved interfaces: $2 d$ elasticity. Int J Numer Methods Eng 63:813-832

10. Gonzalez O (1996) Time integration and discrete Hamiltonian systems. J Nonlinear Sci 6:449-467

11. Gonzalez O (1999) Mechanical systems subject to holonomic constraints: differential-algebraic formulations and conservative integration. Physica D 132:165-174 
12. Gonzalez O (2000) Exact energy and momentum conserving algorithms for general models in nonlinear elasticity. Comput Methods Appl Mech Eng 190:1763-1783

13. Haikal G, Hjelmstad KD (2007) A finite element formulation of non-smooth contact based on oriented volumes for quadrilateral and hexahedral elements. Comput Methods Appl Mech Eng 196:4690-4711

14. Hauret P, LeTallec P (2006) Energy-controlling time integration methods for nonlinear elastodynamics and low-velocity impact. Comput Methods Appl Mech Eng 195:4890-4916

15. Hesch C, Betsch P (2009) A mortar method for energy-momentum conserving schemes in frictionless dynamic contact problems. Int J Numer Methods Eng 77:1468-1500

16. Hesch C, Betsch P (2011) Transient 3d contact problems-NTS method: mixed methods and conserving integration. Comput Mech doi:10.1007/s00466-011-0597-2

17. Hesch C, Betsch P (2010) Transient 3d domain decomposition problems: frame-indifferent mortar constraints and conserving integration. Int J Numer Methods Eng 82:329-358

18. Hintermueller M, Ito K, Kunisch K (2003) The primal-dual active set strategy as a semismooth Newton method. SIAM 13:865-888

19. Hüeber S, Wohlmuth BI (2005) A primal-dual active set strategy for non-linear multibody contact problems. Comput Methods Appl Mech Eng 194:3147-3166

20. Hughes TJR (2000) The finite element method. Dover, NY

21. Krause R, Wohlmuth BI (2000) Nonconforming domain decomposition techniques for linear elasticity. East West J Numer Math 8(3): 177-206

22. Laursen TA (2002) Computational contact and impact mechanics. Springer, Berlin

23. Laursen TA, Chawla V (1997) Design of energy conserving algorithms for frictionless dynamic contact problems. Int $\mathrm{J}$ Numer Methods Eng 40:863-886

24. Laursen TA, Love GR (2002) Improved implicit integrators for transient impact problems-geometric admissibility within the conserving framework. Int J Numer Methods Eng 53:245-274

25. Leimkuhler BJ, Reich S (2004) Simulating Hamiltonian dynamics. Cambridge University Press, Cambridge
26. McDevitt TW, Laursen TA (2000) A mortar-finite element formulation for frictional contact problems. Int J Numer Methods Eng 48:1525-1547

27. Puso MA (2004) A 3d mortar method for solid mechanics. Int J Numer Methods Eng 59:315-336

28. Puso MA, Laursen TA (2004) A mortar segment-to-segment contact method for large deformation solid mechanics. Comput Methods Appl Mech Eng 193:601-629

29. Puso MA, Laursen TA (2004) A mortar segment-to-segment frictional contact method for large deformations. Comput Methods Appl Mech Eng 193:4891-4913

30. Puso MA, Laursen TA, Solberg JM (2008) A segment-to-segment mortar contact method for quadratic elements and large deformations. Comput Methods Appl Mech Eng 197:555-566

31. Simo JC, Tarnow N(1992) The discrete energy-momentum method. Conserving algorithms for nonlinear elastodynamics. $\mathrm{Z}$ angew Math Phys (ZAMP) 43:757-792

32. Simo JC, Tarnow N, Wong KK (1992) Exact energy-momentum conserving algorithms and symplectic schemes for nonlinear dynamics. Comput Methods Appl Mech Eng 100:63-116

33. Wohlmuth BI (2000) A mortar finite element method using dual spaces for the Lagrange multiplier. SIAM J Numer Anal 38(3):989-1012

34. Wohlmuth BI, Krause R (2001) A multigrid methods based on the unconstrained product space arising form motar finite element discretizations. SIAM J Numer Anal 39(1):192-213

35. Wriggers P (2006) Computational contact mechanics, 2nd edn. Springer, Berlin

36. Yang B, Laursen TA (2008) A contact searching algorithm including bounding volume trees applied to finite sliding mortar formulation. Comput Mech 41:189-205

37. Yang B, Laursen TA, Meng X (2005) Two dimensional mortar contact methods for large deformation frictional sliding. Int $\mathrm{J}$ Numer Methods Eng 62:1183-1225

38. Zienkiewicz OC, Taylor RL (2005) The finite element method for solid and structural mechanics, 6th edn. Butterworth Heinemann, London 